\title{
The DES view of the Eridanus supervoid and the CMB cold spot
}
A. Kovács ${ }^{\oplus, 1,2 \star} \dagger$ N. Jeffrey ${ }^{\oplus},{ }^{3,4}$ M. Gatti, ${ }^{5,6}$ C. Chang, ${ }^{7,8}$ L. Whiteway, ${ }^{4}$ N. Hamaus, ${ }^{9}$ O. Lahav, ${ }^{4}$ G. Pollina, ${ }^{9}$ D. Bacon, ${ }^{10}$ T. Kacprzak, ${ }^{11}$ B. Mawdsley, ${ }^{10}$ S. Nadathur, ${ }^{4}$ D. Zeurcher, ${ }^{11}$ J. García-Bellido, ${ }^{12}$ A. Alarcon, ${ }^{13}$ A. Amon, ${ }^{14}$ K. Bechtol, ${ }^{15}$ G. M. Bernstein, ${ }^{6}$ A. Campos, ${ }^{16}$ A. Carnero Rosell, $, 2,17$ M. Carrasco Kind, ${ }^{18,19}$ R. Cawthon, ${ }^{15}$ R. Chen, ${ }^{20}$ A. Choi, ${ }^{21}$ J. Cordero, ${ }^{22}$ C. Davis, ${ }^{14}$ J. DeRose, ${ }^{23,24}$ C. Doux, ${ }^{6}$ A. Drlica-Wagner, ${ }^{7,8,25}$ K. Eckert, ${ }^{6}$ F. Elsner, ${ }^{4}$ J. Elvin-Poole, ${ }^{21,26}$ S. Everett, ${ }^{24}$ A. Ferté, ${ }^{27}$ G. Giannini, ${ }^{5}$ D. Gruen, ${ }^{9,28,29}$ R. A. Gruendl, ${ }^{18,19}$ I. Harrison, ${ }^{22,30}$ W. G. Hartley, ${ }^{31}$ K. Herner, ${ }^{25}$ E. M. Huff, ${ }^{27}$ D. Huterer, ${ }^{32}$ N. Kuropatkin, ${ }^{25}$ M. Jarvis, ${ }^{6}$ P. F. Leget, ${ }^{14}$ N. MacCrann, ${ }^{33}$ J. McCullough, ${ }^{14}$ J. Muir, ${ }^{34}$ J. Myles, ${ }^{14,28,29}$ A. Navarro-Alsina, ${ }^{35}$ S. Pandey, ${ }^{6}$ J. Prat, ${ }^{7}$ M. Raveri, ${ }^{8}$ R. P. Rollins, ${ }^{22}$ A. J. Ross, ${ }^{21}$ E. S. Rykoff, ${ }^{14,29}$ C. Sánchez, ${ }^{6}$ L. F. Secco, ${ }^{6}$ I. Sevilla-Noarbe, ${ }^{36}$ E. Sheldon, ${ }^{37}$ T. Shin, ${ }^{6}$ M. A. Troxel, ${ }^{20}$ I. Tutusaus, ${ }^{38,39}$ T. N. Varga, ${ }^{9,40}$ B. Yanny, ${ }^{25}$ B. Yin, ${ }^{16}$ Y. Zhang,,${ }^{25}$ J. Zuntz, ${ }^{41}$ M. Aguena, ${ }^{17,42}$ S. Allam, ${ }^{25}$ F. Andrade-Oliveira, ${ }^{17,43}$ J. Annis, ${ }^{25}$ E. Bertin, ${ }^{44,45}$ D. Brooks, ${ }^{4}$ D. Burke, ${ }^{14,29}$ J. Carretero, ${ }^{5}$ M. Costanzi, ${ }^{46,47,48}$ L. N. da Costa, ${ }^{17,49}$ M. E. S. Pereira, ${ }^{32}$ T. Davis, ${ }^{50}$ J. De Vicente, ${ }^{36}$ S. Desai, ${ }^{51}$ H. T. Diehl, ${ }^{25}$ I. Ferrero, ${ }^{52}$ B. Flaugher, ${ }^{25}$ P. Fosalba, ${ }^{38,39}$ J. Frieman, ${ }^{8,25}$ E. Gaztañaga, ${ }^{38,39}$ D. Gerdes, ${ }^{32,53}$ T. Giannantonio, ${ }^{54,55}$ J. Gschwend, ${ }^{17,49}$ G. Gutierrez, ${ }^{25}$ S. Hinton, ${ }^{50}$ D. L. Hollowood, ${ }^{24}$ K. Honscheid, ${ }^{21}$ D. James, ${ }^{56}$ K. Kuehn, ${ }^{57,58}$ M. Lima, ${ }^{17,42}$ M. A. G. Maia, ${ }^{17,49}$ J. L. Marshall, ${ }^{59}$ P. Melchior, ${ }^{60}$ F. Menanteau, ${ }^{18,19}$ R. Miquel,,${ }^{5,61}$ R. Morgan, ${ }^{15}$ R. Ogando, ${ }^{17,49}$ F. Paz-Chinchon, ${ }^{18,54}$ A. Pieres, ${ }^{17,49}$ A. A. Plazas, ${ }^{60}$ M. Rodriguez Monroy, ${ }^{36}$ K. Romer, ${ }^{62}$ A. Roodman, ${ }^{14,29}$ E. Sanchez, ${ }^{36}$ M. Schubnell, ${ }^{32}$ S. Serrano, ${ }^{38,39}$ M. Smith, ${ }^{63}$ M. Soares-Santos, ${ }^{32}$ E. Suchyta, ${ }^{64}$ M. E. C. Swanson, ${ }^{18}$ G. Tarle, ${ }^{32}$ D. Thomas, ${ }^{10}$ C.-H. To ${ }^{14,28,29}$ and J. Weller ${ }^{9,40}$

Affiliations are listed at the end of the paper

Accepted 2021 November 7. Received 2021 November 5; in original form 2021 October 5

\begin{abstract}
ABSTRA CT
The Cold Spot is a puzzling large-scale feature in the Cosmic Microwave Background temperature maps and its origin has been subject to active debate. As an important foreground structure at low redshift, the Eridanus supervoid was recently detected, but it was subsequently determined that, assuming the standard $\Lambda$ CDM model, only about 10-20 per cent of the observed temperature depression can be accounted for via its Integrated Sachs-Wolfe imprint. However, $R \gtrsim 100 h^{-1}$ Mpc supervoids elsewhere in the sky have shown ISW imprints $A_{\text {ISW }} \approx 5.2 \pm 1.6$ times stronger than expected from $\Lambda$ CDM $\left(A_{\mathrm{ISW}}=1\right)$, which warrants further inspection. Using the Year-3 redMaGiC catalogue of luminous red galaxies from the Dark Energy Survey, here we confirm the detection of the Eridanus supervoid as a significant underdensity in the Cold Spot's direction at $z<0.2$. We also show, with S/N $\gtrsim 5$ significance, that the Eridanus supervoid appears as the most prominent large-scale underdensity in the dark matter mass maps that we reconstructed from DES Year-3 gravitational lensing data. While we report no significant anomalies, an interesting aspect is that the amplitude of the lensing signal from the Eridanus supervoid at the Cold Spot centre is about 30 per cent lower than expected from similar peaks found in $\mathrm{N}$-body simulations based on the standard $\Lambda \mathrm{CDM}$ model with parameters $\Omega_{\mathrm{m}}=$ 0.279 and $\sigma_{8}=0.82$. Overall, our results confirm the causal relation between these individually rare structures in the cosmic web and in the CMB, motivating more detailed future surveys in the Cold Spot region.
\end{abstract}

Key words: gravitational lensing: weak-surveys - cosmic background radiation.

\section{INTRODUCTION}

^E-mail: akovacs@iac.es

$\dagger$ Juan de la Cierva Fellow.
The Cold Spot (CS) is a large-scale anomaly of about $10^{\circ}$ diameter in the Cosmic Microwave Background (CMB) temperature maps. Centred on $l, b \simeq 209^{\circ},-57^{\circ}$ galactic coordinates, it was first 
Table 1. A collection of the main proposed explanations for the Cold Spot and their current status in the light of existing observational probes.

\begin{tabular}{lcc}
\hline & Cold spot hypothesis & Observational status \\
\hline 1. & Measurement error & excluded, Planck data confirmed it \\
2. & Galactic foreground & excluded, no frequency dependence \\
3. & Sunyaev-Zeldovich effect & excluded, no major low- $z$ cluster \\
4. & Cosmic texture & no evidence from other probes \\
5. & Multiverse signature & no evidence, highly speculative \\
6. & Primordial fluctuation & possible, formally $a \sim 3 \sigma$ CMB fluke \\
7. & Imprint of a supervoid & possible, anomalies in other voids \\
8. & Combined effect from 1-7 & possible, depends on cosmology \\
\hline
\end{tabular}

detected using a spherical harmonic wavelet filtering method (Cruz et al. 2005) in Wilkinson Microwave Anisotropy Probe (WMAP) data set (Bennett et al. 2013), and it was later confirmed in Planck data (Planck Collaboration XXIII 2014). Subsequently, Zhang \& Huterer (2010) and Nadathur et al. (2014) pointed out that the most anomalous nature of the CS is not primarily its coldness at its centre, but rather the combination of a cold interior and a surrounding hot ring. Overall, the CS is equivalent to a $\sim 3 \sigma$ fluctuation in an ensemble of Gaussian CMB skies, thus the null hypothesis of a tailend primordial temperature fluctuation cannot be rejected.

Yet, there has been an active debate about possible physical processes from foreground structures, at low or high redshifts, that might imprint such a spot on the CMB sky (see Table 1 for popular hypotheses). The first proposals for the physical origin of the CS included rather exotic physics, e.g. cosmic textures at $z \approx 1$ (Cruz et al. 2008), without valuable supporting evidence from independent probes (see Vielva 2010, for a detailed review). Another active line of follow-up research was focused on the possible existence of a large underdensity, a supervoid, in the matter distribution in alignment with the CS. The rationale is that, in the presence of a dominant low- $z$ dark energy component, the decaying gravitational potential $(\Phi)$ of a supervoid may generate at least a significant fraction of the observed temperature depression $\left(\Delta T_{0} \approx-150 \mu \mathrm{K}\right.$ in the centre, see Fig. 1 for details) via the late-time Integrated Sachs-Wolfe (ISW) effect (Sachs \& Wolfe 1967). In general, the total ISW shift of the CMB photon temperatures along a direction $\hat{\boldsymbol{n}}$ can be calculated from the time-dependent gravitational potential $(\dot{\Phi} \neq 0)$ based on the line-of-sight integral (other notations may use conformal time)

$$
\frac{\Delta T_{\mathrm{ISW}}}{\bar{T}}(\hat{\boldsymbol{n}})=2 \int_{0}^{z_{\mathrm{LS}}} \frac{a}{H(z)} \dot{\Phi}(\hat{\boldsymbol{n}}, z) \mathrm{d} z,
$$

with $c=1$, scale factor $a=1 /(1+z)$, Hubble parameter $H(z)$, extending to the redshift of last scattering $z_{\mathrm{LS}}$. The gravitational potential $\Phi$ is related to the matter density field $\delta(\boldsymbol{r})$ by the Poisson equation

$\nabla^{2} \Phi(\boldsymbol{r}, z)=\frac{3}{2} H_{0}^{2} \Omega_{\mathrm{m}} \frac{\delta(\boldsymbol{r}, z)}{a}$,

with the Hubble constant $H_{0}$ and matter density parameter $\Omega_{\mathrm{m}}$. In the linear growth approximation, density perturbations grow as $\delta(\boldsymbol{r}, z)=D(z) \delta(\boldsymbol{r})$, where $D(z)$ is the linear growth factor with a typical normalization $D(0)=1$. We note that there are subdominant non-linear Rees-Sciama effects (RS; Rees \& Sciama 1968) that remain at the $\Delta T_{\mathrm{RS}} / \Delta T_{\mathrm{ISW}} \lesssim 10$ per cent level compared to the ISW term (see e.g. Cai et al. 2010, for simulated results).

From a combination of linear growth and the Poisson equation, it follows that $\dot{\Phi}=-H(z)[1-f(z)] \Phi$, where $f=\operatorname{dln} D / \operatorname{dln} a$ is the linear growth rate of structure. Then, one can obtain the following

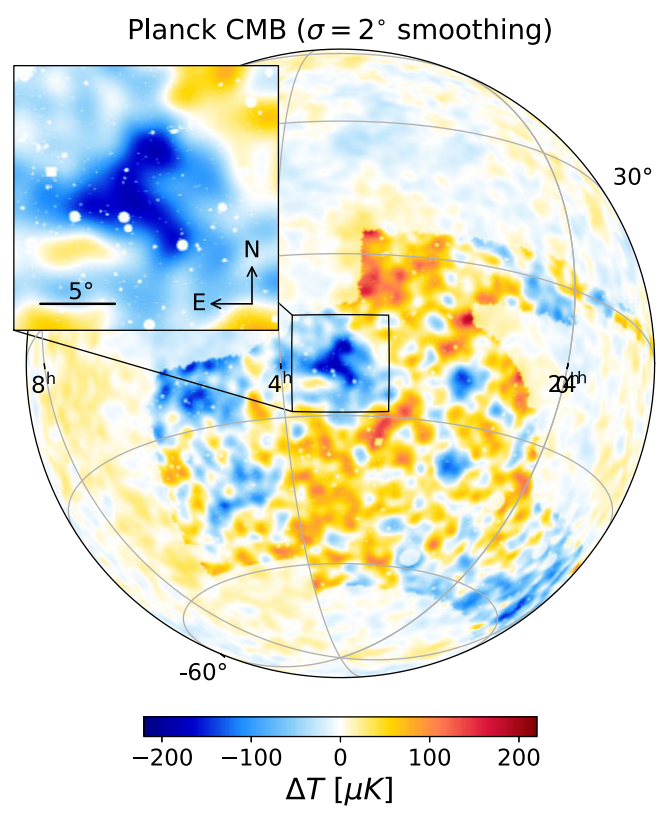

Figure 1. The Planck CMB temperature map (Planck Collaboration VI 2020) is shown centred on the CS with a Gaussian smoothing applied to suppress small-scale fluctuations (using RA and Dec Equatorial coordinates). We highlighted the DES Y3 survey footprint which is the basis of our investigations in this paper. The inset shows a $10^{\circ} \times 10^{\circ}$ zoom-in version of the CS area.

formula for the linear ISW effect that is dominant at large scales:

$\frac{\Delta T_{\mathrm{ISW}}}{\bar{T}}(\hat{\boldsymbol{n}})=-2 \int_{0}^{z_{\mathrm{LS}}} a[1-f(z)] \Phi(\hat{\boldsymbol{n}}, z) \mathrm{d} z$.

Considering the theoretical side of the problem, we note that the ISW signal is sensitive to the underlying cosmology (see e.g. Cai et al. 2014; Beck et al. 2018; Adamek et al. 2020), and this makes the proposed causal relation of a foreground supervoid and the CS an interesting hypothesis. Since the ISW signal is sourced by a suppression of the growth rate $(f<1)$ due to the extra space-stretching effects by dark energy at low redshifts, the details of the measured ISW imprints can constrain the properties of the cosmological constant $(\Lambda)$ in the $\Lambda$-Cold Dark Matter $(\Lambda \mathrm{CDM})$ model.

Note that in order to imprint a strong ISW signal, a supervoid should ideally be located at low redshift where the $[1-f(z)]$ growth suppression factor is the strongest (see equation 3). Earlier in the $\Lambda \mathrm{CDM}$ timeline during the Einstein-de Sitter-like matter-dominated epoch at $z \gtrsim 2$, gravitational growth, and cosmic expansion are in balance $(f \approx 1)$, implying constant gravitational potentials $(\dot{\Phi} \approx 0)$ and $\Delta T_{\text {ISW }} \approx 0$. Based on equation ( 3 ), the second requirement for a strong ISW imprint is a large fluctuation in $\Phi$ (see e.g. Nadathur, Hotchkiss \& Crittenden 2017), which is also more easily met in the low $z$ range where the cosmic web features bigger density fluctuations.

The first expectation was that an exceptionally deep $\left(\delta_{0} \approx-1\right)$ and very large $\left(R \gtrsim 200 h^{-1} \mathrm{Mpc}\right)$ supervoid at $z \approx 1$ could, in principle, imprint the CS via the ISW effect (Inoue \& Silk 2006, 2007; Inoue, Sakai \& Tomita 2010). However, a sensible reasoning to exclude any supervoid explanation was that in the standard theory of peaks for Gaussian random fields (Bardeen et al. 1986) the probability of the formation of a supervoid capable of imprinting a CS-like profile via the ISW effect is practically zero (corresponding to a $\gtrsim 5 \sigma$ density fluctuation) in $\Lambda$ CDM (Nadathur et al. 2014). The CS itself is a $\sim 3 \sigma$ 


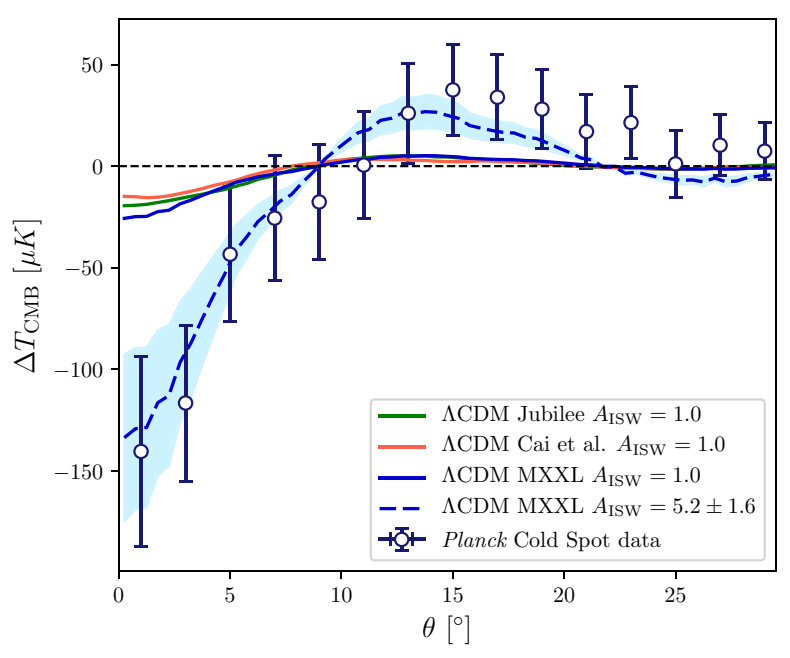

Figure 2. A comparison of the observed CS data and the $\Delta T_{\text {ISW }}$ profiles aligned with 'coldest spots' in three different $\mathrm{N}$-body simulations assuming a $\Lambda$ CDM model $\left(A_{\text {ISW }}=1, \Delta T_{0} \approx-20 \mu \mathrm{K}\right)$. For the MXXL simulation, we also show an enhanced ISW signal by re-scaling the $\Lambda$ CDM template with $A_{\text {ISW }} \approx 5.2 \pm 1.6$ that was determined from the observational analysis of several other supervoids, including DES Y3 and BOSS DR12 data.

fluctuation in Gaussian CMB map statistics of cold spots and thus hypothesizing such an unlikely supervoid makes no sense in solving the problem itself.

Alternatively, the CS might be composed of a moderate negative fluctuation in the primordial CMB plus a small negative $\Delta T_{\text {ISW }}$ contribution, rather than it being entirely a primordial fluctuation or entirely an ISW imprint. Smaller and/or shallower voids at lower redshift may partially contribute to the observed temperature depression, but such structures are incapable of explaining the total CS profile in a standard $\Lambda$ CDM model (Naidoo, Benoit-Lévy \& Lahav 2016).

On the observational front, the existence of significant voids at $z$ $\gtrsim 0.3$ has been excluded with high confidence (Bremer et al. 2010; Granett, Szapudi \& Neyrinck 2010), in line with the theoretical expectations discussed above. As a culmination of an extensive search, the relatively shallow $\left(\delta_{0} \approx-0.2\right)$, but certainly extended $(R$ $\approx 200 h^{-1} \mathrm{Mpc}$ ) Eridanus supervoid was discovered at $z \approx 0.15$ in the direction of the CS (see e.g. Szapudi et al. 2015, and Section 2 below). Assuming a baseline $\Lambda \mathrm{CDM}$ model, there is a consensus about the corresponding ISW imprint of supervoids with parameters consistent with the above observationally determined values (see e.g. Nadathur et al. 2014; Finelli et al. 2015; Marcos-Caballero et al. 2016; Naidoo et al. 2016; Naidoo, Benoit-Lévy \& Lahav 2017). As shown in Fig. 2, the expected central ISW imprint is of order $\Delta T_{0} \approx-20 \mu \mathrm{K}$, in accordance with the 'coldest spot' in the simulated Jubilee (Watson et al. 2014), Millennium XXL (Angulo et al. 2012), and (Cai et al. 2010) ISW maps using the same definition and wavelet filtering technique on mock ISW maps (Kovács 2018; Kovács et al. 2020).

Therefore, among others, Nadathur et al. (2014) and Mackenzie et al. (2017) concluded that a causal relation is certainly plausible, but the Eridanus supervoid can only explain about 10-20 per cent of the observed CS profile $\left(\Delta T_{0} \approx-150\right.$ cent $\left.\mu \mathrm{K}\right)$ in the $\Lambda \mathrm{CDM}$ model.

None the less, an important further aspect is that the observed amplitude of the ISW signal $\left(A_{\mathrm{ISW}} \equiv \Delta T_{\mathrm{obs}} / \Delta T_{\Lambda \mathrm{CDM}}\right)$ is often significantly higher from supervoids than expected in the concordance model $\left(A_{\text {ISW }}=1\right)$. Such excess ISW signals were first found by
Granett, Neyrinck \& Szapudi (2008) using luminous red galaxies (LRG) from the Sloan Digital Sky Survey (SDSS) data set. Followup measurements and simulation analyses then determined that the observed signal is in about $\sim 3 \sigma$ tension with the $\Lambda$ CDM model expectations (see e.g. Nadathur, Hotchkiss \& Sarkar 2012; Flender, Hotchkiss \& Nadathur 2013; Hernández-Monteagudo \& Smith 2013; Ilić, Langer \& Douspis 2013; Aiola, Kosowsky \& Wang 2015).

To further test the claimed ISW anomalies, Kovács et al. (2017) used photo- $z$ catalogues of LRGs from the Dark Energy Survey (DES Collaboration 2016) Year-1 data set (DES Y1) and reported an excess signal, similar to the original SDSS detection by Granett et al. (2008). This analysis was extended to the DES Year-3 data set and the excess ISW signals were confirmed (Kovács et al. 2019). These findings were crucial, because they independently detected ISW anomalies using a different part of the sky.

In combination with the BOSS results using similarly defined supervoids (Kovács 2018), the ISW amplitude from BOSS DR12 and DES Y3 data is $A_{\text {ISW }} \approx 5.2 \pm 1.6$ in the $0.2<z<0.9$ redshift range. Note that this excess ISW amplitude appears to be consistent with the enhancement that would be necessary to fully explain the CS as an ISW imprint from the Eridanus supervoid, as shown in Fig. 2. An obvious question to ask: is this a coincidence, or the two ISW-like anomalies concerning supervoids are related?

In this paper, we approach this problem from a different perspective. Unlike ISW measurements, recent analyses of the CMB lensing imprints of the anomalous supervoids showed no excess signal neither using BOSS (Cai et al. 2017; Raghunathan et al. 2020) nor DES Y1 data (Vielzeuf et al. 2021), which might provide new insights. Along similar lines, here we use the state-of-the-art dark matter mass maps reconstructed using the DES Y3 data set (Jeffrey et al. 2021) to study the gravitational lensing signal of the Eridanus supervoid. In particular, we explore how special it is in the $4100 \mathrm{deg}^{2}$ DES Y3 footprint and how its shape and amplitude compare to $\Lambda \mathrm{CDM}$ expectations (see e.g. Higuchi \& Inoue 2018).

The paper is organized as follows. In Section 2, we provide a historical account of cosmographic analyses in the CS area, and also describe the DES data sets. We present our observational methods and results in Section 3, and then compare our findings to simulated results in Section 4. Finally, Section 5 presents a discussion of our main results including our conclusions.

\section{MAPPING THE COLD SPOT AREA}

\subsection{Existing results: from galaxy maps to cosmic flows}

The first evidence for an underdensity aligned with the CS was presented by Rudnick, Brown \& Williams (2007) by studying a catalogue of radio galaxies in the NRAO VLA Sky Survey (NVSS). However, no redshift information was available for the void candidate, and the significance of the detection was disputed (Smith \& Huterer 2010).

Targeted pencil beam surveys (Granett et al. 2010 and Bremer et al. 2010) found no evidence for a significant underdensity between redshifts of $0.5<z<0.9$, but their galaxy counts were consistent with a void at $z<0.3$. In addition, the analysis of the 2-Micron All-Sky Survey Extended Source Catalogue (2MASS XSC; Jarrett et al. 2000) galaxy distribution by Francis \& Peacock (2010) showed a shallow underdensity of large angular size around the CS. Rassat, Starck \& Dupé (2013) confirmed the presence of this low- $z$ void in the reconstructed 2MASS ISW maps. Manzotti \& Dodelson (2014) found that any late time ISW-RS imprints that might be responsible 
for the CS are very likely to originate at $z<0.3$, motivating a detailed examination of this range.

Then, Finelli et al. (2015) analysed the low- $z$ WISE-2MASS galaxy catalogue (Kovács \& Szapudi 2015) that combines measurements of two all-sky surveys in the infrared, the Wide-Field Infrared Survey Explorer (WISE; Wright et al. 2010) and the Point Source Catalogue of the 2-Micron All-Sky Survey (2MASS; Skrutskie et al. 2006). They identified a $\sim 20$ per cent underdensity $\left(\delta_{0} \approx-0.2\right)$ in the direction of the $\mathrm{CS}$ with $\theta \approx 20^{\circ}$ angular size that corresponds to about $R \approx 200 h^{-1} \mathrm{Mpc}$ physical radius, i.e. a rare density fluctuation given the combination of size and underdensity.

Along similar lines, Szapudi et al. (2015) matched the WISE2MASS galaxy data set to a $1300 \mathrm{deg}^{2}$ area with Pan-STARRS1 (PS1 Kaiser 2004) data, adding optical colours for each object. For the resulting catalogue, photometric redshifts were estimated and the line-of-sight galaxy density profile was analysed in the redshift range $z<0.3$. Further evidence was found for a shallow but extended $\operatorname{supervoid}\left(\delta_{0} \approx-0.2, R \approx 200 h^{-1} \mathrm{Mpc}\right)$ centred on the $\mathrm{CS}$, indicating a roughly spherical structure in combination with the constraints on it transverse size by Finelli et al. (2015).

After the discovery of this underdensity called the Eridanus supervoid, Kovács \& García-Bellido (2016) analysed the 2MASS photo- $z$ catalogue (Bilicki et al. 2014, 2MPZ) and found that the supervoid is elongated and its extent in the line-of-sight might be larger than $R \approx 200 \mathrm{~h}^{-1} \mathrm{Mpc}$, extending to the lowest redshifts.

A further development was the dedicated $2 \mathrm{CSz}$ spec- $z$ survey of about 7000 galaxies in the CS region at $z<0.4$. Mackenzie et al. (2017) identified four smaller voids at different redshifts which suggests substructure for the Eridanus supervoid. However, they also identified a similar underdensity in the line of sight in their control sample elsewhere in the sky (not observed by DES). They then argued that the absence of a CS-like pattern in alignment with this other system of voids suggests that the Eridanus supervoid is not a special structure in the low- $z$ Universe and therefore there is no causal relation with the CS. Alternatively, the overall volume of the Eridanus supervoid in 3D may be larger than that of the underdensity in the control field by Mackenzie et al. (2017) depending on the largescale environments around the measured lines of sight. This second hypothesis is also supported by Courtois et al. (2017) who analysed their Cosmicflows-3 data set and found that the 'Cold Spot Repeller' is the largest basin of repulsion in the $z \approx 0.1$ cosmic web, closely aligned with the CS. Therefore, the Eridanus supervoid appears to be a rare underdensity in a full $3 \mathrm{D}$ view and further investigations are needed to determine its connections to the CS; including this analysis using DES data.

\subsection{Motivation: the lensing imprint of voids}

The weak lensing information from DES Y3 data is a key novelty in the problem of the CS and the Eridanus supervoid. In a wider context, it helps us to contribute to an established line of research on the mass distribution in cosmic voids, including possible precision tests of alternative cosmological models in void environments (see e.g. Clampitt, Cai \& Li 2013; Cai, Padilla \& Li 2015; Baker et al. 2018; Cautun et al. 2018; Davies, Cautun \& Li 2019).

Unlike clusters, groups, and filaments, cosmic voids cause a demagnification effect and therefore correspond to local minima in the lensing convergence $(\kappa)$ maps, estimated from the matter density field $\delta(r, \theta)$ via projection as

$\kappa(\theta)=\frac{3 H_{0}^{2} \Omega_{m}}{2 c^{2}} \int_{0}^{r_{\max }} \delta(r, \theta) \frac{\left(r_{\max }-r\right) r}{r_{\max }} \mathrm{d} r$, where $r$ denotes a comoving distance to the sources, and $r_{\max }$ determines the maximum distance considered in the projection.

Several real-world detections of the lensing signal from multiple voids using stacking methods have already been reported (Melchior et al. 2014; Clampitt \& Jain 2015; Gruen et al. 2016; Sánchez et al. 2017; Brouwer et al. 2018; Fang et al. 2019), including CMB lensing analyses (Cai et al. 2017; Raghunathan et al. 2020; Hang et al. 2021a; Vielzeuf et al. 2021). These observations are supported and complemented by signal-to-noise optimization efforts and tests of the signal shape given different void definitions using N-body simulations (see e.g. Cautun, Cai \& Frenk 2016; Davies, Cautun \& Li 2018; Davies et al. 2021).

However, the detection of the weak lensing effect of an individual void has been considered a great challenge due to the significant measurement uncertainties (see e.g. Krause et al. 2013), except in the case of the largest voids with $R \gtrsim 100 h^{-1} \mathrm{Mpc}$ radius (Amendola, Frieman \& Waga 1999). Therefore, the Eridanus supervoid is a good candidate for such a measurement given its low redshift and approximate $R \approx 200 \mathrm{~h}^{-1} \mathrm{Mpc}$ radius.

Higuchi \& Inoue (2018) used an N-body simulation with a lensing convergence map and found an expected significance of $\mathrm{S} / \mathrm{N} \gtrsim 4$ for a weak lensing signal from such an extended low- $z$ underdensity assuming a standard $\Lambda$ CDM model. Higuchi \& Inoue (2019) also showed that measurable decreasing trends are expected in the nonGaussian peak statistics in $\kappa$ maps towards the largest underdense regions such as the Eridanus supervoid. These recent findings from simulations provide motivation for our observational analysis. Our main methods are the following:

(i) we first measure a line-of-sight galaxy density profile at the CS using LRGs selected from DES data.

(ii) we then rely on the gravitational lensing convergence $(\kappa)$ maps, reconstructed from DES cosmic shear measurements, and follow up on the detection of the Eridanus supervoid using dark matter mass maps, and compare the results to $\mathrm{N}$-body simulations.

\subsection{DES Y3 data: Luminous red galaxies}

We mapped the CS region using data products from the first three years (Y3) of the Dark Energy Survey (DES, DES Collaboration 2016; Abbott et al. 2018). DES is a six-year survey that covers approximately $4100 \mathrm{deg}^{2}$ sky area of the South Galactic Cap. Mounted on the Cerro Tololo Inter-American Observatory (CTIO) four metre Blanco telescope in Chile, the 570 megapixel Dark Energy Camera (DECam, Flaugher et al. 2015) images the field in grizY filters.

The raw images were processed by the DES Data Management (DESDM) team (Sevilla et al. 2011; Morganson et al. 2018). We adopted the empirically constructed DES Y3 survey mask in our analysis, which excludes potentially contaminated pixels e.g. in the close proximity of bright stars. For the full details of the DES Y3 data set, we refer the readers to Sevilla-Noarbe et al. (2021).

To estimate the line-of-sight galaxy density profile aligned with the CS, we used an LRG sample from the first three years of observations. This red-sequence MAtched-filter Galaxy Catalog (redMaGiC; Rozo et al. 2016) is a catalogue of photometrically selected LRGs, based on the red-sequence MAtched-filter Probabilistic Percolation (redMaPPer) cluster finder algorithm (Rykoff et al. 2014). We utilized the $\mathrm{redMaGiC}$ sample that spans the $0.2<z<0.7$ range, because of its exquisite photometric redshifts, namely $\sigma_{z} /(1+z)$ $\approx 0.02$, and a $4 \sigma$ redshift outlier rate of $r_{\text {out }} \simeq 1.41$ per cent. The resulting galaxy sample has an approximately constant comoving 
space density $\bar{n} \approx 10^{-3} h^{3} \mathrm{Mpc}^{-3}$ (high density sample, brighter than $0.5 L_{*}$ ).

The great photo- $z$ precision allows an accurate and robust reconstruction of cosmic void environments, and such a redMaGiC galaxy sample has been used in a series of DES void analyses including weak lensing and ISW measurements (see e.g. Kovács et al. 2017; Sánchez et al. 2017; Fang et al. 2019; Vielzeuf et al. 2021).

The galaxy clustering properties of the latest $\mathrm{Y} 3 \mathrm{redMaGiC}$ data set are presented by Pandey et al. (2021). In the context of possible remnant systematic effects in this sample (see e.g. DES Collaboration 2021, for further details), we note that 3222 DES voids, identified from the Y3 redMaGiC data, did show correlations with DES mass map features (Jeffrey et al. 2021). This indicates that genuine cosmic voids are detected from this catalogue.

\subsection{DES Y3 data: dark matter mass maps}

Complementing galaxy catalogues, the mass maps are weighted projections of the density field (primarily dark matter) in the foreground of the observed galaxies. Following the DES Y3 mass map reconstruction analysis presented in detail by Jeffrey et al. (2021), we consider mass maps in HEALPix format with resolution $N_{\text {side }}=1024$ (Gorski et al. 2005) based on four slightly different reconstruction methods; each is a maximum a posteriori estimate with a different model for the prior probability of the map:

(i) The first method considered is the direct inversion of the shear field, also known as the Kaiser-Squires (KS) method (Kaiser \& Squires 1993), followed by a smoothing of small angular scales.

(ii) The second method uses a prior on the B-modes of the map, imposing that the reconstructed convergence field must be purely an E-mode map (null B-mode prior); this method also includes smoothing at small scales.

(iii) The third method, the Wiener filter, uses a Gaussian prior distribution for the underlying convergence field.

(iv) Lastly, the GLIMPSE method implements a sparsity prior in wavelet (starlet) space (Lanusse et al. 2016), which can be interpreted as a physical model where the matter field is composed of a superposition of spherically symmetric haloes.

All methods are implemented on the celestial sphere to accommodate the large sky coverage of the DES Y3 data. The mass maps were reconstructed using the DES Y3 shear catalogue Gatti et al. (2021) of 100204026 galaxies in $4143 \mathrm{deg}^{2}$, building upon the Y3 Gold catalogue (Sevilla-Noarbe et al. 2021) and using the METACALIBRATION algorithm (Huff \& Mandelbaum 2017; Sheldon \& Huff 2017), which infers the galaxy ellipticities starting from noisy images of the detected objects in the $r, i, z$ bands.

Map-level tests against various systematics regarding the parent DES galaxy catalogues showed no significant remnant contamination. Focusing on large scales, here we take the DES Y3 mass maps as inputs and present an additional application in cosmographic analyses (see Jeffrey et al. 2018, 2021, for further details about reconstruction methods and mass map properties).

\section{METHODS AND RESULTS}

\subsection{A line-of-sight density profile}

Without considering lensing information, this first part of our analysis aims at measuring and testing the galaxy density field from the DES data in the direction of the CS, and test the consistency with previous state-of-the-art results (see e.g. Kovács \& García-Bellido 2016;
Mackenzie et al. 2017). Following similar DES analyses presented by Chang et al. (2018) and Jeffrey et al. (2021), the redMaGiC galaxy catalogue was projected into the $2 \mathrm{D}$ slices of $\Delta r=100 \mathrm{~h}^{-1} \mathrm{Mpc}$ along the line of sight. This thickness corresponds to the approximate photo- $z$ errors of the redMaGiC galaxies that allows the robust identification of voids (see Sánchez et al. 2017, for details).

At $0.07<z<0.45$, galaxy density contrasts $\left(\rho_{\mathrm{g}} / \bar{\rho}_{\mathrm{g}}-1=\delta_{\mathrm{g}}\right)$ are measured in 10 tomographic slices aligned with the CS at RA, Dec $\approx$ $48.3^{\circ},-20.4^{\circ}$. Galaxies were counted within an aperture of $2.5^{\circ}$ of the void centre. This corresponds to approximately half the angular size of the CS, allowing a more direct analysis of the deepest parts of the supervoid.

To facilitate comparisons to the previous $2 \mathrm{MPZ}$ and $2 \mathrm{CSz}$ surveys in the CS area that used different galaxy tracers with different linear bias, we converted the galaxy density value to matter density $\left(\delta_{\mathrm{m}}=\right.$ $\delta_{\mathrm{g}} / b_{\mathrm{g}}$ ) in all cases. We note that the large and shallow (super)voids detected by photo- $z$ surveys such as DES are well described by the linear bias model; they naturally trace the largest scales where the linear bias approximation is expected to hold (see Pollina et al. 2017, 2019, for previous simulated and DES results).

Estimated from a combination of clustering and lensing 2-point correlation functions (Prat et al. 2021), the linear galaxy bias for the DES Y3 redMaGiC sample is about $b_{\mathrm{g}} \approx 1.74 \pm 0.12$ at our redshifts of interests (a slight redshift dependence is neglected in this analysis). The linear galaxy bias values for the $2 \mathrm{MPZ}$ galaxy catalogue (Bilicki et al. 2014) are $b_{\mathrm{g}} \approx 1.18 \pm 0.03$ at $z<0.08$ and $b_{\mathrm{g}} \approx 1.52 \pm 0.03$ at $0.08<z<0.3$, as determined by Alonso et al. (2015). The linear bias of the $2 \mathrm{CSz}$ galaxies changes gradually from $b_{\mathrm{g}} \approx 1.35$ at $z \approx 0.1$ to about $b_{\mathrm{g}} \approx 2.0$ for galaxies approaching $z \approx$ 0.4 (Mackenzie et al. 2017). We linearly interpolated between these measured $b_{\mathrm{g}}$ values, and used them for our conversion from galaxy to matter density fluctuations.

As shown in Fig. 3, the matter density contrasts estimated from DES data at the different redshifts are remarkably consistent not only with the 2MPZ photo- $z$ data in their overlapping range, but also with the presumably more accurate $2 \mathrm{CSz}$ spec- $z$ results. This agreement further suggests that our analysis is certainly not affected by possible remnant systematic problems concerning the DES Y3 redMaGiC catalogue (DES Collaboration 2021).

With the DES data, we confirmed the previously reported deepest part $(\delta \approx-0.25$ ) of the Eridanus supervoid at $z \approx 0.15$, observed a slight overdensity $(\delta \lesssim 0.1)$ at $z \approx 0.2$, and also detected two smaller voids at higher redshifts. Our redMaGiC LRG data contain few galaxies in the $z<0.1$ range and therefore we cannot provide a detailed comparison with existing measurements. In this generally underdense low $z$ environment (see $2 \mathrm{MPZ}$ data points), the intervening overdense structure at $z \approx 0.06$ (detected only in more accurate spec- $z$ data) is one of the outer filamentary features rooted in the Horologium supercluster, as noted previously by Kovács \& García-Bellido (2016) in their cosmographical analysis.

Considering the $z>0.45$ redshift range, we note that previous lineof-sight analyses of galaxy counts by Granett et al. (2010) and Bremer et al. (2010) excluded the possibility of significant voids aligned with the CS (while their results were consistent with an underdensity at $z<0.3$ where later the Eridanus supervoid was detected). For completeness, we did check the $0.45<z<0.7$ range using our DES redMaGiC galaxies and, as expected, found no significant voids or superclusters (see Appendix A for details).

Our reconstruction of the matter density field at the CS from the DES Y3 data set confirms that the Eridnaus supervoid is among the largest known underdensities in the observable Universe. A deeper 


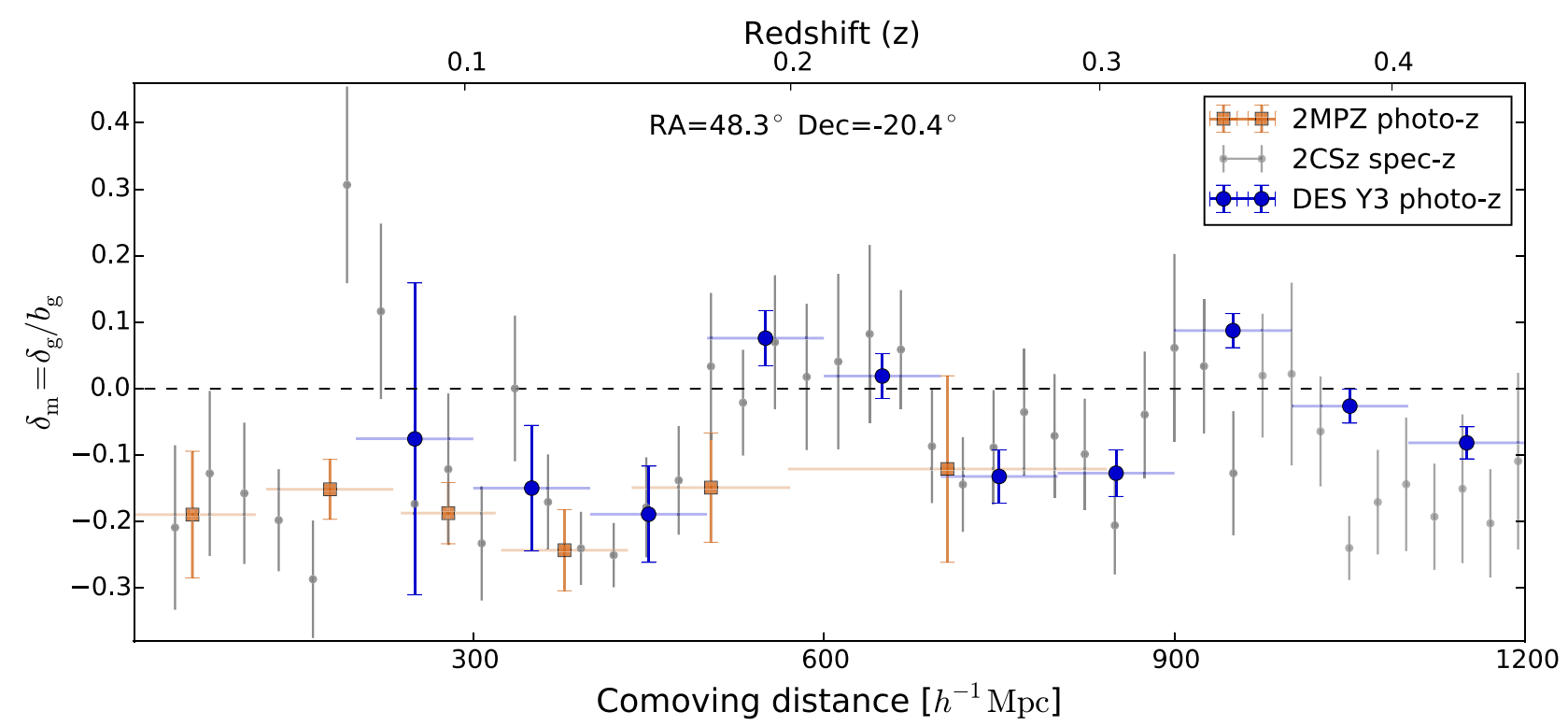

Figure 3. The line-of-sight matter density profiles are compared for different surveys of the CS direction. We converted galaxy density to matter density using the independently determined linear galaxy bias $\left(b_{\mathrm{g}}\right)$ values for each tracer data set. The DES results from redMaGiC galaxies feature a consistent density profile when compared to $2 \mathrm{MPZ}$ (photo- $z$ ) and $2 \mathrm{CSz}$ (spec- $z$ ) data. We also observe two previously seen smaller voids at redshifts higher than the main supervoid at $z$ $<0.2$, which may also contribute to the overall ISW imprints of the supervoid.

understanding of these large-scale structures is of great interest in cosmology (see e.g. Shimakawa et al. 2021).

\subsection{Mass map filtering strategies}

The DES Y3 weak lensing convergence $(\kappa)$ maps are exceptionally rich sources of cosmological information. For instance, additional information on the growth of structure and the clumpiness of the matter distribution might be extracted by analysing their higher order moments (Gatti et al. 2020), or their non-Gaussian peak statistics may also be measured with machine learning techniques to complement traditional 2-point function analyses (see e.g. Ribli et al. 2019; Zürcher et al. 2021).

In this analysis, we are guided to ignore the information encoded in their small-scale patterns, and rather test their largest scales for three reasons. First, the existing evidence on the dimensions of the Eridanus supervoid suggests that its angular size is about $\theta \approx 20^{\circ}$ as a consequence of its very low redshift and large physical size with about $R \approx 200 h^{-1} \mathrm{Mpc}$. Secondly, to test previous claims that this supervoid is not a particularly special underdensity in the low- $z$ Universe (see e.g. Mackenzie et al. 2017) we wish to know if this region is of special significance considering the total $4100 \mathrm{deg}^{2}$ DES Y3 survey area.

Thirdly, we expect from the Poisson equation, conveniently expressed in Fourier space as $\Phi(\boldsymbol{k}, z) \sim \delta(\boldsymbol{k}, z) / \boldsymbol{k}^{2}$ using a wave vector $\boldsymbol{k}$, that the characteristic fluctuations in the gravitational potential appear on much larger scales than in the density field due to the $\boldsymbol{k}^{-2}$ factor. Since these perturbations in $\Phi$ are the actual sources of the ISW signal, it is important to focus on their reconstruction.

Along these lines, we followed three similar strategies to highlight and probe the largest scales in the DES Y3 mass maps:

(i) as the simplest proxy for tracing large-scale patterns in the gravitational potential, we applied a Gaussian smoothing to the mass maps with $\sigma=10^{\circ}$. (ii) as an alternative method, we filtered the mass maps in spherical harmonic space and kept only the $\ell<10$ modes which correspond to the largest angular scales.

(iii) we calculated the projected gravitational potential $\psi$ from the $\kappa$ maps following a $\kappa_{\ell m}=-\frac{1}{2} \ell(\ell+1) \psi_{\ell m}$ transformation which also effectively highlights the largest scales in the map.

Our reconstructed large-scale patterns in the DES Y3 KS mass map are shown in Fig. 4. We report a striking visual correlation between the location of the CS and the largest negative density fluctuation in the maps, regardless of the methodology to probe the largest scales. We also observed a generally overdense environment further away from the CS region. The significance of these correlations is estimated from more detailed analysis below.

Overall, this finding certainly confirms previous detections of the Eridanus supervoid from an independent new tracer of large-scale structure. Moreover, it also indicates that this supervoid is indeed a special underdensity in the low- $z$ cosmic web, and reinforces the hypothesis of causal correlations with the CS.

\subsection{Void lensing profiles at the cold spot}

An important consideration is that the CS is located close to the edge of the DES survey area as shown in Fig. 4. We note that the originally designed DES footprint was in fact modified (during the survey planning stage) to fully include the CS area. Therefore, the observed DES Y3 data set does include a complete $\theta \lesssim 15^{\circ}$ disc around the nominal CS centre $\left(\mathrm{RA}, \mathrm{Dec} \approx 48.3^{\circ},-20.4^{\circ}\right.$ ) which allows a detailed view of the large-scale structure of DES galaxies in this direction. However, this limitation also means that any radially averaged measurement beyond $\theta \approx 15^{\circ}$ will necessarily result in a less complete reconstruction due to an increasing fraction of masked pixels beyond the edge of the survey (most importantly in the direction of the top left-hand corner of the insets in Fig. 4).

We quantified the visually compelling correlations seen in Fig. 4 by measuring the tangential $\kappa$ and $\psi$ profiles in $\Delta \theta=2^{\circ}$ bins centred on 

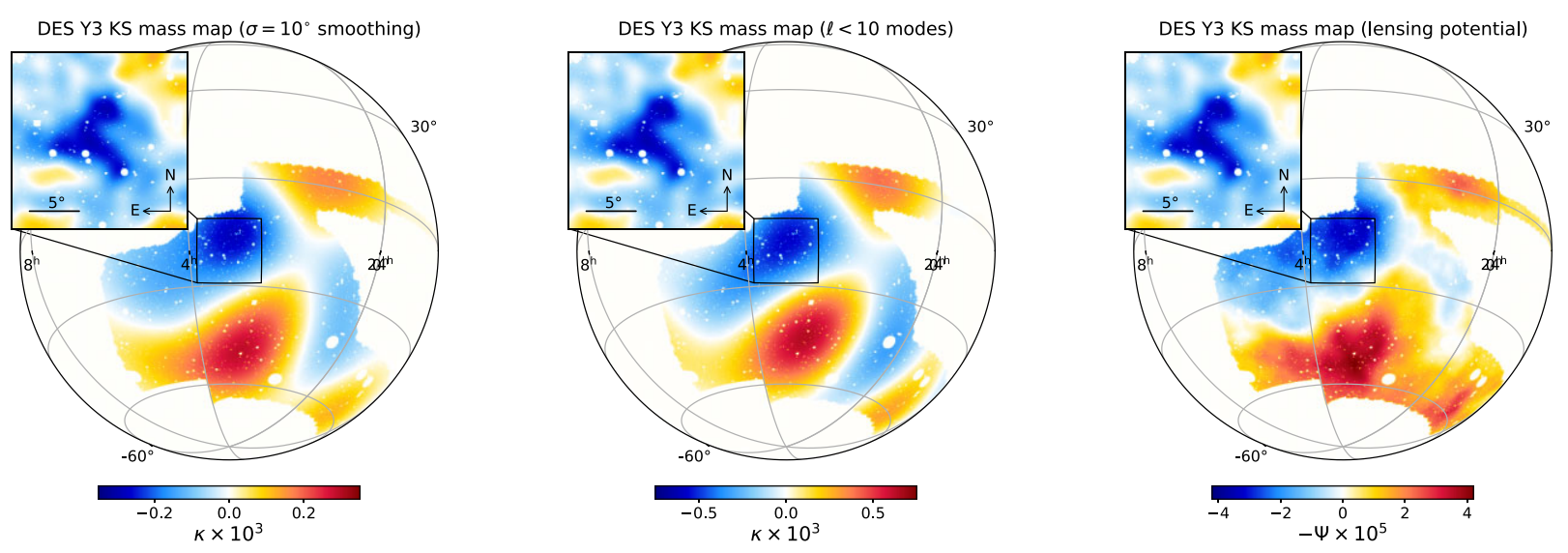

Figure 4. Using HEALPix tools, the left-hand panel shows the DES KS mass map with a $\sigma=10^{\circ}$ Gaussian smoothing applied to highlight fluctuations at the largest scales. The middle panel displays our results for a mass map with $\ell<10$ modes only. On the right, we show the projected gravitational potential ( $\psi$ ) that we calculated from the $\kappa$ map. Our maps consistently show that the largest under-density in the DES Y3 data set is closely aligned with the CS, corresponding to a prominent low- $z$ supervoid. As in Fig. 1, the inset in all panels shows the Planck CMB temperature map in the CS region.

the CS. We estimated the uncertainties of this detection by measuring the radial $\kappa$ profile in 200 random locations in the DES Y3 mass maps. The resulting covariance matrix $\mathrm{C}$ showed strong off-diagonal contributions, especially at the innermost $4-5$ bins, which we take into account in our analysis. We then evaluated a $\chi^{2}$ statistic and calculated the signal-to-noise ratio compared to a null detection as $\mathrm{S} / \mathrm{N}=\sqrt{\chi_{0}^{2}-N_{\text {bins }}}$ with $\chi_{0}^{2}=\kappa_{\text {data }} C^{-1} \kappa_{\text {data }}$, where $C^{-1}$ is the inverse of the covariance matrix from our randoms and $\kappa_{\text {data }}$ are the measured data points in the radial profile.

We compared our results from different versions of the DES Y3 mass map based on the Kaiser-Squires (KS), Wiener filter, and Null-B reconstruction methods. We note that, by construction, the GLIMPSE method is not adequate to accurately recover the largest scales and therefore we do not use the related mass map in our main analyses. For completeness, we nevertheless detected an underdensity aligned with the CS in the GLIMPSE map as well, although with a lower amplitude than in the other three versions.

\subsubsection{Gaussian smoothing $\left(\sigma=10^{\circ}\right)$}

In Fig. 5, we present our results in the case of the Gaussian smoothing applied to the mass maps. All maps show a consistently negative and gradually fading $\kappa$ imprint which extends to about $\theta \approx 20^{\circ}$, in line with previous findings from the WISE-2MASS galaxy map (Finelli et al. 2015). Testing the relation of fluctuations in mass and light, we also measured the projected galaxy density field at the CS using the DES redMaGiC galaxies. We found an approximately linear relationship between $\delta_{\mathrm{g}}$ and $\kappa_{\mathrm{g}}$, as expected based on previous results (Fang et al. 2019; Pollina et al. 2019).

Considering only the innermost radial bin at $\theta<2^{\circ}$, we found $\mathrm{S} / \mathrm{N}$ $\approx 2.1$ as a signal-to-noise ratio. Using the data from all radial bins up to $\theta \approx 40^{\circ}$, we report an $\mathrm{S} / \mathrm{N} \approx 7.2$ detection of an underdensity in the fiducial Kaiser-Squires mass map. While this $\mathrm{S} / \mathrm{N}$ analysis was based on the full covariance matrix $\mathrm{C}$ (including bin-to-bin correlations), the error bars shown in Fig. 5 were estimated from the diagonal elements for demonstration. Given these uncertainties, the Wiener filtered and Null B-mode results are fully consistent with the KS signal, which strengthens our detection.

We note that a slightly overdense 'compensation' region $(\kappa>0)$ is also detected around the central underdensity $(\kappa<0)$. These data points also contribute to the $\mathrm{S} / \mathrm{N}$ that we estimate considering their

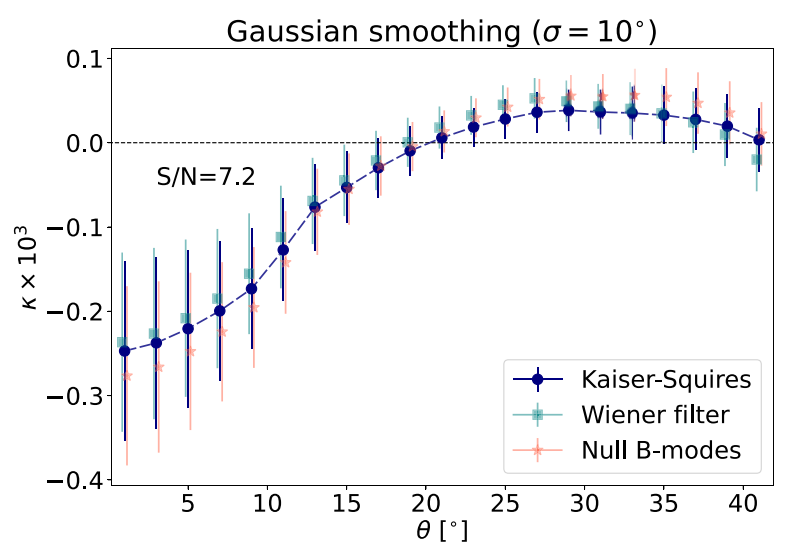

Figure 5. Tangential $\kappa$ profiles centred on the CS. In the case of the KS map, the detection significance of the measured $\kappa$ profile is $\mathrm{S} / \mathrm{N} \approx 7.2$ compared to a null signal. We compare our three different mass map versions and report good consistency among them at all radii.

covariance with the inner part of the profile. At large $\theta$, the lensing signal does approach zero as expected. See also Fig. 4 for a visual impression of the surroundings of the CS area.

\subsubsection{Large-scale modes $(\ell<10)$}

In Fig. 6, we then compare the shape of the $\kappa$ profile in the different DES mass map versions if only the largest scales are considered with $\ell<10$. The detection significance is $\mathrm{S} / \mathrm{N} \approx 2.1$ for the first bin at the centre, whilst the full profile yields an $\mathrm{S} / \mathrm{N} \approx 6.1$ detection.

Given the errors, we again report great consistency between different mass map versions, and also with the results from Gaussian smoothing. The underdense central part, the over-dense compensation region, and the convergence to zero signal are again clearly detected using these differently filtered maps.

We note that the amplitude of the $\kappa$ profiles from the $\ell<10$ maps are higher compared to the results from Gaussian smoothing. This is the consequence of the slightly different mass map filtering techniques that we applied to highlight the largest scales, but both sets of Wiener, Null B-mode, and KS maps are internally consistent. 


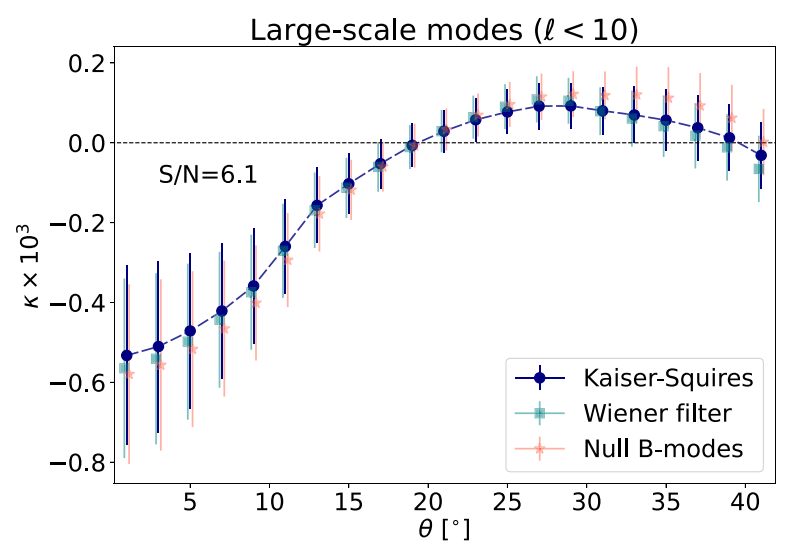

Figure 6. Convergence $(\kappa)$ profiles measured from the $\ell<10$ filtered version of the maps to study the actual large-scale modes. When comparing different mass maps, we again see good consistency throughout the full extent of the profile, including the compensation zone at $\theta \gtrsim 20^{\circ}$.

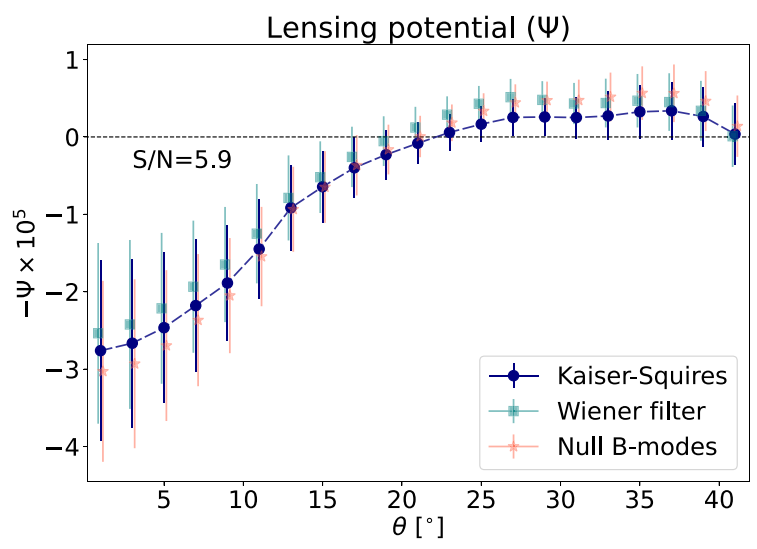

Figure 7. Tangential $\psi$ profiles measured from the projected gravitational potential maps that we calculated from the original $\kappa$ maps without smoothing or filtering. We again report consistent results in the full extent of the profile from different mass maps.

\subsubsection{Lensing potential $(\psi)$}

In Fig. 7, we illustrate how the reconstructed large-scale gravitational potential at the CS changes when different map versions are used. As in the previous two cases, we observe a consistent profile shape and amplitude. An underdensity is detected with $\mathrm{S} / \mathrm{N} \approx 2.3$ considering only the first bin, but the overall detection significance reaches $\mathrm{S} / \mathrm{N}$ $\approx 5.9$ considering the full extent of the profile. Given the errors, the measured profiles from different mass map versions show consistent results, including the compensation zone beyond the zero-crossing at about $\theta \approx 20^{\circ}$.

From these tests, we also observed that the transverse size of the under-density extends beyond the actual CS region, and other surrounding voids are also expected to contribute to this large fluctuation in the gravitational potential. We note that another large void with $R \approx 250 h^{-1} \mathrm{Mpc}$ line-of-sight size, that Jeffrey et al. (2021) detected in alignment with a significant $\kappa<0$ region in the DES Y3 mass map at $\mathrm{RA}, \operatorname{Dec} \approx 41.2^{\circ},-12.2^{\circ}$, is also expected to contribute to the gravitational potential fluctuation $\left(\theta \approx 7^{\circ}\right.$ from the CS centre).

This finding suggests that the actually deepest part of the Eridanus supervoid may be outside the central CS region, but the overall largescale fluctuation in the $\psi$ map is very accurately centred on the CS,

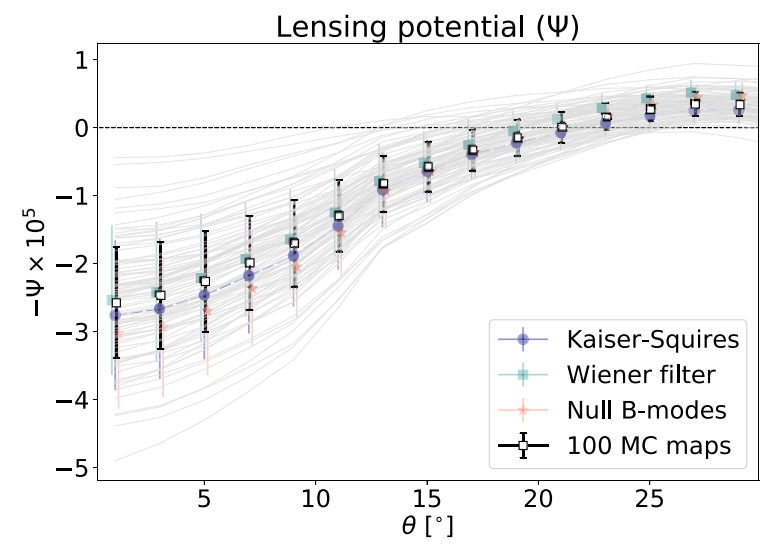

Figure 8. Tangential profiles from 100 independent Monte Carlo samples from the posterior $p(\kappa \mid \gamma)$ using the Wiener posterior (light grey curves) with fixed void position are compared to our main results that are based on maximum a posteriori mass maps from different reconstruction methods. Though the error bars represent different uncertainties, the mean and standard deviation (black markers) of this ensemble is in great agreement with our baseline analyses in the full extent of the profile.

and this is what drives the expected ISW signal. We leave the more detailed analysis of the substructure of the Eridanus supervoid for future work, possibly including upcoming DES data releases and novel spectroscopic data sets.

\subsection{Monte Carlo sampling}

To evaluate the uncertainty associated with the lensing potential profile at fixed position, we target the posterior probability $p(\psi \mid \gamma, \mathrm{RA}, \mathrm{Dec})$ given lensing data $\gamma$. We generate independent Monte Carlo (MC) samples from this $\psi$ posterior using samples from the posterior of possible mass maps $p(\kappa \mid \gamma)$. For each sample of the posterior probability of the map $\kappa$, we evaluate the lensing potential $\psi$ and measure the radial profile for the fixed RA, Dec of the CS.

For the mass map posterior samples, we use the same Gaussian prior as used by the Wiener filter. The Wiener filter is equivalent to the mean of all possible posterior samples (in addition to being the maximum of the posterior distribution). Each posterior sample is a constrained realization (Hoffman \& Ribak 1991; Zaroubi et al. 1995) and each appears as a full-sky mass map with the expected power spectrum and masked regions in painted. Unobserved regions of the sky are lightly constrained by data and will therefore fluctuate heavily between samples. To generate $\kappa$ realizations drawn from $p(\kappa \mid \gamma)$ we use the DANTE ${ }^{1}$ package (Kodi Ramanah, Lavaux \& Wandelt 2019) with settings matching those described by Jeffrey et al. (2021).

In Fig. 8, we present the results from the analysis of $100 \mathrm{MC}$ mass map samples. The uncertainties that we estimated from the MC sampling are comparable to the errors we estimated from randomization of the void centre in the DES footprint but their actual meaning is slightly different. The MC errors characterize our knowledge on the amplitude and shape of the reconstructed void profile, whereas the errors from randomization correspond to a detection significance compared to a null hypothesis of no void detection.

Given these errors, we found that the mean profile calculated from this ensemble of profiles is not identical to the baseline maximum a posteriori Wiener filter map result. However, the two results are fully

${ }^{1}$ https://github.com/doogesh/dante 
consistent with each other, and also with the other two mass map versions Kaiser-Squires and Null B-modes.

\section{VOID LENSING IN SIMULATIONS}

Considering all the evidence presented, we report a robust $\mathrm{S} / \mathrm{N} \gtrsim$ 5 detection of a supervoid from the DES Y3 mass maps aligned with the CS. Importantly, this finding is fully consistent with the expectation of an $\mathrm{S} / \mathrm{N} \gtrsim 4$ detection from an Eridanus-like supervoid based on the simulation analysis by Higuchi \& Inoue (2018).

\subsection{Methodology}

To better assess the consistency of the observed lensing signal with $\Lambda \mathrm{CDM}$ expectations, we also analysed convergence maps from $\mathrm{N}$ body simulations. We used a set of full-sky mock lensing maps (Takahashi et al. 2017) obtained for source redshifts $z \lesssim 1.4$, in consistency with the range of DES Y3 source galaxies. Initial conditions were generated using the $2 \mathrm{LPTIC}$ code (Crocce, Pueblas \& Scoccimarro 2006) and the N-body simulation used L-GADGET2 (Springel et al. 2005) with cosmological parameters consistent with the WMAP 9-year results: $\Omega_{\mathrm{m}}=0.279, \sigma_{8}=0.82, \Omega_{\mathrm{b}}=0.046, n_{\mathrm{s}}$ $=0.97, h=0.7$ (see Hinshaw et al. 2013, for details).

The average matter power spectra of the simulations agree with the revised HALOFIT (Takahashi et al. 2012) predictions within 5 per cent for $k<1 h \mathrm{Mpc}^{-1}$ at $z<1$ and for $k<0.8 h \mathrm{Mpc}^{-1}$ at $z$ $<3$. A multiple plane ray-tracing algorithm (GRayTrix; Hamana et al. 2015) was used to estimate the values of the convergence fields for the simulation snapshots, and $\kappa$ maps are provided in the form of HEALPix maps with different resolutions, including $N_{\text {side }}=4096$ which we used for our tests.

\subsection{Consistency tests}

To model our DES Y3 analysis, we downgraded the mock $\kappa$ maps to $N_{\text {side }}=1024$, and converted them to $\psi$ maps by applying the $\kappa_{\ell m}=$ $-\frac{1}{2} \ell(\ell+1) \psi_{\ell m}$ transformation. We considered five different mock $\Lambda \mathrm{CDM}$ realizations, and also analysed each octant separately in the five full-sky maps. With this strategy, we accounted for the $4100 \mathrm{deg}^{2}$ survey window of the observed DES data (using a less complicated mask than in observations, but identical map resolutions), and thereby built an ensemble of $5 \times 8=40$ DES Y3-like simulations to compare to. We also checked that the simulations feature similar fluctuations in the maps compared to the DES data, and that using lower resolution maps does not change the results.

In each of the DES Y3-like octant mocks, we identified the location of the HEALP ix pixel with the highest value of $\psi$, which corresponds to the approximate centre of the largest projected underdensity in the map. As in the DES Y3 analysis, we then measured the tangential profiles around these most significant peaks in the $\psi$ maps, and determined their mean and standard deviation.

As shown in Fig. 9, we found that, in the centre of the CS $(\theta$ $\lesssim 5^{\circ}$ ), the lensing signal from the Eridanus supervoid is about 30 per cent lower than typical results from the $\Lambda$ CDM mocks. The significance of the observed discrepancy is at the $2 \sigma$ level, with a $\psi_{0}$ $\approx(2.8 \pm 1.2) \times 10^{-5}$ central value for DES Y 3 and $\psi_{0} \approx(5.3 \pm 0.8)$ $\times 10^{-5}$ measured for our mocks. At $\theta \approx 3^{\circ}$ and $\theta \approx 5^{\circ}$, we also found a $1.7 \sigma$ and a $1.2 \sigma$ lower-than-expected signal, respectively, followed by consistent signal strength in the rest of the profile.

We stress that this is a frequentist analysis; the cosmological parameters $\Omega_{\mathrm{m}}=0.279$ and $\sigma_{8}=0.82$ are not varied in our mock realizations. Therefore, the $2 \sigma$ discrepancy that we found should be

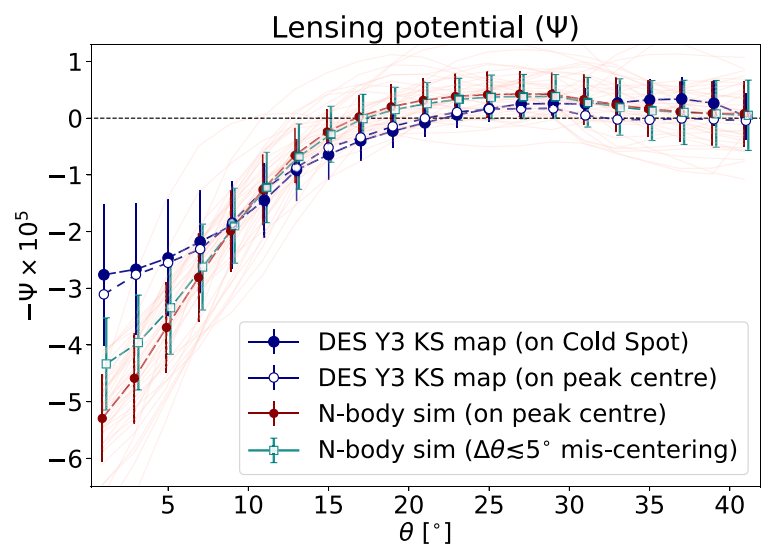

Figure 9. A comparison of lensing signals from supervoids, identified as large-scale peaks in $\psi$ maps, using DES Y3 data (dark blue) and 40 mock realizations (pale red curves). The red points and error bars show the mean and standard deviation of the 40 simulated analyses, if the profiles are centred on the actual peak of the potential fluctuations. The discrepancy with observations cannot be resolved even when a random $\Delta \theta \lesssim 5^{\circ}$ peak miscentring is applied in simulations (empty squares). It is also demonstrated that the actual peak in DES Y3 data is very closely aligned with the nominal CS position, and measuring the $\psi$ profile around this observed peak (empty circles) does not significantly change the DES Y3 results.

interpreted as the likelihood of detecting such a low lensing signal given the specific $\Lambda \mathrm{CDM}$ model parameters, and also considering the field-to-field fluctuations in the simulated measurements.

\subsection{Discussion and interpretation}

While this mismatch is not highly significant, we considered possible explanations. First, we tested the expected role of mis-centring in the identification of the largest peak in the $\psi$ maps. As demonstrated in Fig. 9, a randomly assigned $\Delta \theta \lesssim 5^{\circ}$ shift of the peak position in mock profile measurements could reduce the $\sim 2 \sigma$ tension to the even more tolerable $1.3 \sigma$ level (and also results in a slight widening of the lensing profiles at large radii, i.e. similar to the DES results). Then, we also identified the position of the large-scale peak in the DES Y3 $\psi$ map, finding RA, Dec $\approx 45.0^{\circ},-18.5^{\circ}$, i.e. only about $\Delta \theta \approx 3.8^{\circ}$ from the nominal CS centre at RA, Dec $\approx 48.3^{\circ},-20.4^{\circ}$. We measured the tangential profile in the DES Y $3 \psi$ map around this location, and found an approximately 10 per cent stronger signal in the centre (see Fig. 9). Therefore, the observed mis-centring does not explain all of the discrepancy (but it may contribute to it), assuming that the DES mass maps are correct.

We also considered that a lower lensing signal might come from imperfections in the observational reconstruction of the underlying potential $\psi$. The performance of the DES Y3 mass mapping methods was validated using a single realization of the Takahashi et al. (2017) simulations that we also analysed. In that example, Jeffrey et al. (2021) found that the power spectrum of the reconstructed mass map simulations was underpredicted by $\sim 10-30$ per cent at the largest scales $(\ell \lesssim 15)$ compared the true noiseless mock convergence field. In a more realistic analysis, a noisy realization of the same underlying $\kappa$ map was analysed, and no significant bias was found in the KS and Null B-mode map reconstructions. We also highlight here that the DES survey window provides an incomplete map of the surroundings of the wider CS region (see Fig. 4), and thus the reconstruction of the large-scale potential may also be imperfect, both in terms of centring and in overall amplitude. 
We note that the large-scale modes may vary quite significantly in different realizations due to cosmic variance. While the role of these variations was not extensively tested in the DES Y3 mass map analyses (Jeffrey et al. 2021), a consistent suppression of large-scale modes in DES Y3 mass map reconstructions could in principle result in lower amplitudes at the large-scale peaks. In our measurements and mock analyses, we made an attempt to minimize such possible biases by setting the $\ell \leq 3$ spherical harmonic modes to zero in the $\psi$ map. At the expense of losing some of the signal, this simple highpass filtering removes supersurvey modes from the lensing potential map $\psi$ that a DES Y3-like $4100 \mathrm{deg}^{2}$ survey is unable to probe. In turn, the strongest local peaks in the full-sky $\psi$ map's octants became more comparable to the cut-sky DES Y3 maps.

Overall, the flattened lensing profile observed in DES data may well be a combined effect from the above sources of imperfections concerning the data (mis-centring, nearby survey edge, suppression of large-scale power in the map reconstructions), but we stress that the observed discrepancy remains at the moderate $2 \sigma$ level.

Finally, we also contemplated that the lower-than-expected lensing signal in the direction of the CS centre is due to a genuine physical effect, since there are intriguing precedents for similar findings in cosmology. For example, Leauthaud et al. (2017) reported from their BOSS $\times$ CFHTLenS galaxy-galaxy lensing measurements that the observed lensing signal is $\sim 20-40$ per cent lower than expected based on the autocorrelation of the galaxy sample. Then, Lange et al. (2021) determined that this tension does not significantly depend on the mass of haloes in the $10^{13.3}-10^{13.9} h^{-1} \mathrm{M}_{\odot}$ range and no significant scale-dependence is seen in the $0.1 h^{-1} \mathrm{Mpc}<r<60 h^{-1} \mathrm{Mpc}$ range. These results can exclude some proposed small-scale phenomena as explanations, such as baryonic effects or insufficient halo occupation modelling.

Considering cosmic voids, we highlight that the CMB lensing imprint of DES Y1 voids was also found slightly lower than expected with about $A_{\kappa}=\kappa_{\text {obs }} / \kappa_{\text {th }} \approx 0.8$ (Vielzeuf et al. 2021). While the significance of this DES Y1 result was only moderate, Hang et al. (2021a) analysed a larger sample of similarly defined voids and superclusters using the Legacy Survey photo- $z$ catalogue (Dey et al. 2019), and reported $A_{\kappa} \approx 0.811 \pm 0.057$, i.e. $3.3 \sigma$ lower signal than expected from a standard $\Lambda$ CDM model.

As in the case of the ISW excess signals discussed in Section 1, it is important to note that not all void samples show anomalously low lensing signals (see e.g. Cai et al. 2017; Raghunathan et al. 2020), and therefore more work is needed to settle this debate, including this analysis of the CS area and the Eridanus supervoid. Taken at face value, a lower-than-expected lensing signal could in principle be a consequence of a faster low- $z$ expansion rate and a related stronger decay of the gravitational potentials $(\dot{\Phi}<0)$ than assumed in the baseline $\Lambda$ CDM model (see equations 1 and 3), i.e. low lensing and strong ISW signals are not inconsistent.

We note that the $S_{8}=\sigma_{8} \sqrt{\Omega_{\mathrm{m}} / 0.3} \approx 0.79$ lensing parameter from the mocks, given the $\Omega_{\mathrm{m}}=0.279$ and $\sigma_{8}=0.82$ parameters, is consistent with the main DES Y3 result $S_{8} \approx 0.776 \pm 0.017$ (DES Collaboration 2021). This implies that while the $\Omega_{\mathrm{m}}$ and $\sigma_{8}$ parameters may differ, the overall lensing amplitude is expected to agree at the 2 per cent level, which provides a good basis for comparison.

\section{SUMMARY AND CONCLUSIONS}

In this paper, we investigated the CMB CS region using the Year-3 data set from the Dark Energy Survey (DES Collaboration 2016). To advance the mapping of the low $z$ Eridanus supervoid aligned
Table 2. The estimated $\mathrm{S} / \mathrm{N}$ of a supervoid detection in the KS mass map for the three different map filtering strategies. We compare the detection significances for the most central bin only, and if all 21 bins are used.

\begin{tabular}{lccc}
\hline Map version: & $\psi$ & $\ell<10$ & $\sigma=10^{\circ}$ \\
\hline Bins 1-21 & 5.9 & 6.1 & 7.2 \\
Bin 1 only & 2.3 & 2.1 & 2.1 \\
\hline
\end{tabular}

with the CS, we used the redMaGiC catalogue of LRGs. As a key innovation, we also analysed state-of-the-art weak lensing data in the form of dark matter mass maps reconstructed from the DES Y3 data set (Jeffrey et al. 2021).

As demonstrated in Fig. 3, we first measured the line-of-sight galaxy density profile in the direction of the CS centre. In consistency with previous galaxy surveys (see e.g. Mackenzie et al. 2017), we provided more evidence for the existence of the Eridanus supervoid $\left(R \approx 200 h^{-1} \mathrm{Mpc}, \delta_{0} \approx-0.2\right)$ from the distribution of DES Y3 redMaGiC galaxies at $z \lesssim 0.2$.

We then presented a robust $\mathrm{S} / \mathrm{N} \gtrsim 5$ detection of the Eridanus supervoid from the reconstructed DES Y3 mass maps (see Table 2), in line with the expectations from related $\mathrm{N}$-body simulation analyses by Higuchi \& Inoue (2018). We found no significant difference in the lensing profiles when considering different mass map reconstruction methods (Kaiser-Squires, Wiener filter, Null B-modes). Also, our detection is stable when changing our methodology to highlight the largest scales from the DES $\kappa$ maps $\left(\sigma=10^{\circ}\right.$ Gaussian smoothing, $\ell<10$ filtering, lensing potential $\psi$ ). In particular, our findings confirmed that the Eridanus supervoid is the most prominent largescale underdensity in the $4100 \mathrm{deg}^{2}$ survey footprint mapped by the DESY3 data set (see Fig. 4), further suggesting a causal connection with the CS.

Finally, we tested the lensing signal's amplitude in the direction of the CS. We looked for the strongest large-scale peaks in the lensing potential map $\psi$ in N-body simulations (Takahashi et al. 2017), and compared our DES Y3 results to the resulting ensemble of mock supervoid profiles. Interestingly, we found that the observed lensing imprint of the Eridanus supervoid is $\sim 30$ per cent lower than expected from measurements of the largest voids found in mocks based on the $\Lambda \mathrm{CDM}$ model. We noted that this discrepancy is observed at the moderate $\sim 2 \sigma$ significance level (frequentist analysis), restricted only to the CS centre at $\theta \lesssim 5^{\circ}$ (see Fig. 9 for details).

We none the less considered three possible explanations. Using mock lensing potential $(\psi)$ maps, we determined that the observed discrepancy cannot be fully resolved by assuming a random $\Delta \theta \lesssim 5^{\circ}$ peak mis-centring, but it could reduce the tension to the $1.3 \sigma$ level. We also argued that a consistent underestimation of the large-scale modes in the DES Y3 mass map reconstruction process might also explain such a discrepancy, and this possibility is consistent with existing mass mapping analyses in simulations (Jeffrey et al. 2021).

As a third option, we also considered that the low lensing signal at the CS is due to a genuine physical effect. We provided examples for $\sim 20-40$ per cent lower-than-expected lensing amplitudes measured from overdensities (Leauthaud et al. 2017) and also from voids (Vielzeuf et al. 2021; Hang et al. 2021a). If the low- $z$ growth rate of structure in supervoids is even more suppressed than in $\Lambda \mathrm{CDM}$, that leads to shallower gravitational potentials, weaker lensing effects, and a stronger ISW signal. Note that this interesting possibility is consistent with the excess ISW signals observed from a statistical analysis of $R \gtrsim 100 h^{-1}$ Mpc supervoids (Kovács et al. 2019). 
In the context of the CS, a hitherto unknown alternative cosmological model might also provide explanation for the large enhancement that would be needed to explain its deep $\Delta T_{0} \approx-150 \mu \mathrm{K}$ central temperature depression as an ISW imprint, if the underlying model of dark energy is not the cosmological constant (see e.g. Beck et al. 2018; Kovács et al. 2020). Therefore, possible relations to the Hubble constant tension (see e.g. Di Valentino et al. 2021) and the $S_{8}$ problem (see e.g. Heymans et al. 2021; Secco et al. 2021) should also be explored in greater details.

We note that not all data sets and methodologies agree on the detection of such excess ISW signals (see e.g. Hang et al. 2021a), and the claimed tensions often remain undetected using smaller voids (Nadathur \& Crittenden 2016) or two-point correlation functions (Hang et al. 2021b). Nevertheless, recent ISW measurements using the eBOSS quasar catalogue (Ross et al. 2020), covering the $0.8<$ $z<2.2$ range, also showed ISW anomalies at redshifts higher than before (see Kovács et al. 2021, for details), further suggesting an alternative growth history in supervoid environments.

In the light of these findings, the imprint of superstructures in the $\mathrm{CMB}$ remains an interesting unsolved problem in cosmology. Future releases of the DES data and other weak lensing and galaxy surveys such as HSC, KiDS, Euclid, eBOSS, and DESI will certainly help to converge to a solution, together with a more precise mapping of the CS including CMB polarization data (Kang et al. 2020).

\section{ACKNOWLEDGEMENTS}

This paper has gone through internal review by the DES collaboration. AK has been supported by a Juan de la Cierva Incorporación fellowship with project number IJC2018-037730-I, and funding for this project was also available in part through SEV-2015-0548 and AYA2017-89891-P. The authors thank an anonymous referee whose insights improved the clarity of the paper.

Funding for the DES Projects has been provided by the U.S. Department of Energy, the U.S. National Science Foundation, the Ministry of Science and Education of Spain, the Science and Technology Facilities Council of the United Kingdom, the Higher Education Funding Council for England, the National Center for Supercomputing Applications at the University of Illinois at Urbana-Champaign, the Kavli Institute of Cosmological Physics at the University of Chicago, the Center for Cosmology and Astro-Particle Physics at the Ohio State University, the Mitchell Institute for Fundamental Physics and Astronomy at Texas A\&M University, Financiadora de Estudos e Projetos, Fundação Carlos Chagas Filho de Amparo à Pesquisa do Estado do Rio de Janeiro, Conselho Nacional de Desenvolvimento Científico e Tecnológico and the Ministério da Ciência, Tecnologia e Inovação, the Deutsche Forschungsgemeinschaft and the Collaborating Institutions in the Dark Energy Survey.

The Collaborating Institutions are Argonne National Laboratory, the University of California at Santa Cruz, the University of Cambridge, Centro de Investigaciones Energéticas, Medioambientales y Tecnológicas-Madrid, the University of Chicago, University College London, the DES-Brazil Consortium, the University of Edinburgh, the Eidgenössische Technische Hochschule (ETH) Zürich, Fermi National Accelerator Laboratory, the University of Illinois at UrbanaChampaign, the Institut de Ciències de l'Espai (IEEC/CSIC), the Institut de Física d'Altes Energies, Lawrence Berkeley National Laboratory, the Ludwig-Maximilians Universität München and the associated Excellence Cluster Universe, the University of Michigan, NFS's NOIRLab, the University of Nottingham, The Ohio State University, the University of Pennsylvania, the University of Portsmouth, SLAC National Accelerator Laboratory, Stanford
University, the University of Sussex, Texas A\&M University, and the OzDES Membership Consortium.

Based in part on observations at Cerro Tololo Inter-American Observatory at NSF's NOIRLab (NOIRLab Prop. ID 2012B-0001; PI: J. Frieman), which is managed by the Association of Universities for Research in Astronomy (AURA) under a cooperative agreement with the National Science Foundation.

The DES data management system is supported by the National Science Foundation under Grant Numbers AST-1138766 and AST-1536171. The DES participants from Spanish institutions are partially supported by MICINN under grants ESP2017-89838, PGC2018-094773, PGC2018-102021, SEV-2016-0588, SEV-20160597, and MDM-2015-0509, some of which include ERDF funds from the European Union. IFAE is partially funded by the CERCA program of the Generalitat de Catalunya. Research leading to these results has received funding from the European Research Council under the European Union's Seventh Framework Program (FP7/2007-2013) including ERC grant agreements 240672, 291329, and 306478. We acknowledge support from the Brazilian Instituto Nacional de Ciência e Tecnologia (INCT) do e-Universo (CNPq grant 465376/2014-2).

The manuscript has been authored by Fermi Research Alliance, LLC under Contract No. DE-AC02-07CH11359 with the U.S. Department of Energy, Office of Science, Office of High Energy Physics.

\section{DATA AVAILABILITY}

Galaxy catalogues and lensing data will become publicly available as part of the release of the DES Year- 3 products. ${ }^{2}$ The N-body simulations and the corresponding $\kappa$ maps by Takahashi et al. (2017) are publicly available. ${ }^{3}$ The Planck CMB temperature maps that we used to visualize the Cold Spot region are also public. ${ }^{4}$

The data underlying this article including the reconstructed void lensing profiles will be shared on reasonable request to the corresponding author, in line with the relevant DES data policy.

\section{REFERENCES}

Abbott T. M. C. et al., 2018, ApJS, 239, 18

Adamek J., Rasera Y., Corasaniti P. S., Alimi J.-M., 2020, Phys. Rev. D, 101, 023512

Aiola S., Kosowsky A., Wang B., 2015, Phys. Rev. D, 91, 043510

Alonso D., Salvador A. I., Sánchez F. J., Bilicki M., García-Bellido J., Sánchez E., 2015, MNRAS, 449, 670

Amendola L., Frieman J. A., Waga I., 1999, MNRAS, 309, 465

Angulo R. E., Springel V., White S. D. M., Jenkins A., Baugh C. M., Frenk C. S., 2012, MNRAS, 426, 2046

Baker T., Clampitt J., Jain B., Trodden M., 2018, Phys. Rev. D, 98, 023511

Bardeen J. M., Bond J. R., Kaiser N., Szalay A. S., 1986, ApJ, 304, 15

Beck R., Csabai I., Rácz G., Szapudi I., 2018, MNRAS, 479, 3582

Bennett C. L. et al., 2013, ApJS, 208, 20

Bilicki M., Jarrett T. H., Peacock J. A., Cluver M. E., Steward L., 2014, ApJS, 210, 9

Bremer M. N., Silk J., Davies L. J. M., Lehnert M. D., 2010, MNRAS, 404, L69

Brouwer M. M. et al., 2018, MNRAS, 481, 5189

Cai Y.-C., Cole S., Jenkins A., Frenk C. S., 2010, MNRAS, 407, 201

\footnotetext{
${ }^{2}$ https://www.darkenergysurvey.org/the-des-project/data-access/

${ }^{3}$ http://cosmo.phys.hirosaki-u.ac.jp/takahasi/allsky_raytracing/

${ }^{4}$ https://www.cosmos.esa.int/web/planck
} 
Cai Y.-C., Li B., Cole S., Frenk C. S., Neyrinck M., 2014, MNRAS, 439, 2978

Cai Y.-C., Padilla N., Li B., 2015, MNRAS, 451, 1036

Cai Y.-C., Neyrinck M., Mao Q., Peacock J. A., Szapudi I., Berlind A. A., 2017, MNRAS, 466, 3364

Cautun M., Cai Y.-C., Frenk C. S., 2016, MNRAS, 457, 2540

Cautun M., Paillas E., Cai Y.-C., Bose S., Armijo J., Li B., Padilla N., 2018, MNRAS, 476, 3195

Chang C. et al., 2018, MNRAS, 475, 3165

Clampitt J., Jain B., 2015, MNRAS, 454, 3357

Clampitt J., Cai Y.-C., Li B., 2013, MNRAS, 431, 749

Courtois H. M., Tully R. B., Hoffman Y., Pomarède D., Graziani R., Dupuy A., 2017, ApJ, 847, L6

Crocce M., Pueblas S., Scoccimarro R., 2006, MNRAS, 373, 369

Cruz M., Martínez-González E., Vielva P., Cayón L., 2005, MNRAS, 356, 29

Cruz M., Martínez-González E., Vielva P., Diego J. M., Hobson M., Turok N., 2008, MNRAS, 390, 913

Davies C. T., Cautun M., Li B., 2018, MNRAS, 480, L101

Davies C. T., Cautun M., Li B., 2019, MNRAS, 490, 4907

Davies C. T., Paillas E., Cautun M., Li B., 2021, MNRAS, 500, 2417

DES Collaboration, 2016, MNRAS, 460, 1270

DES Collaboration, 2021, preprint (arXiv:2105.13549)

Dey A. et al., 2019, AJ, 157, 168

Di Valentino E. et al., 2021, Class. Quantum Gravity, 38, 153001

Fang Y., Hamaus N., Jain B., Pandey S., DES Collaboration, 2019, MNRAS, 490, 3573

Finelli F., García-Bellido J., Kovács A., Paci F., Szapudi I., 2015, MNRAS, 455,1246

Flaugher B. et al., 2015, AJ, 150, 150

Flender S., Hotchkiss S., Nadathur S., 2013, J. Cosmol. Astropart. Phys., 2013, 13

Francis C. L., Peacock J. A., 2010, MNRAS, 406, 14

Gatti M. et al., 2020, MNRAS, 498, 4060

Gatti M. et al., 2021, MNRAS, 504, 4312

Górski K. M., Hivon E., Banday A. J., Wandelt B. D., Hansen F. K., Reinecke M., Bartelmann M., 2005, ApJ, 622, 759

Granett B. R., Neyrinck M. C., Szapudi I., 2008, ApJ, 683, L99

Granett B. R., Szapudi I., Neyrinck M. C., 2010, ApJ, 714, 825

Gruen D. et al., 2016, MNRAS, 455, 3367

Hamana T., Sakurai J., Koike M., Miller L., 2015, PASJ, 67, 34

Hang Q., Alam S., Cai Y.-C., Peacock J., 2021a, MNRAS, 507, 510

Hang Q., Alam S., Peacock J. A., Cai Y.-C., 2021b, MNRAS, 501, 1481

Hernández-Monteagudo C., Smith R. E., 2013, MNRAS, 435, 1094

Heymans C. et al., 2021, A\&A, 646, A140

Higuchi Y., Inoue K. T., 2018, MNRAS, 476, 359

Higuchi Y., Inoue K. T., 2019, MNRAS, 488, 5811

Hinshaw G. et al., 2013, ApJS, 208, 19

Hoffman Y., Ribak E., 1991, ApJ, 380, L5

Huff E., Mandelbaum R., 2017, ApJ, preprint (arXiv:1702.02600)

Ilić S., Langer M., Douspis M., 2013, A\&A, 556, A51

Inoue K. T., Silk J., 2006, ApJ, 648, 23

Inoue K. T., Silk J., 2007, ApJ, 664, 650

Inoue K. T., Sakai N., Tomita K., 2010, ApJ, 724, 12

Jarrett T. H., Chester T., Cutri R., Schneider S., Skrutskie M., Huchra J. P., 2000, AJ, 119, 2498

Jeffrey N. et al., 2018, MNRAS, 479, 2871

Jeffrey N. et al., 2021, MNRAS, 505, 4626

Kaiser N., 2004, in Oschmann J. M., Jr, ed., Proc. SPIE Conf. Ser. Vol. 5489, Ground-based Telescopes. SPIE, Bellingham, p. 11

Kaiser N., Squires G., 1993, ApJ, 404, 441

Kang J. H. et al., 2020, in Zmuidzinas J., Gao J.-R., eds, Proc. SPIE Conf. Ser. Vol. 11453, Millimeter, Submillimeter, and Far-Infrared Detectors and Instrumentation for Astronomy X. SPIE, Bellingham, p. 114532D

Kodi Ramanah D., Lavaux G., Wandelt B. D., 2019, MNRAS, 490, 947

Kovács A., 2018, MNRAS, 475, 1777

Kovács A., García-Bellido J., 2016, MNRAS, 462, 1882

Kovács A., Szapudi I., 2015, MNRAS, 448, 1305
Kovács A., Sánchez C., García-Bellido J., the DES collaboration, 2017, MNRAS, 465, 4166

Kovács A., Sánchez C., García-Bellido J., the DES collaboration, 2019, MNRAS, 484, 5267

Kovács A., Beck R., Szapudi I., Csabai I., Rácz G., Dobos L., 2020, MNRAS, 499, 320

Kovács A., Beck R., Smith A., Rácz G., Csabai I., Szapudi I., 2021, MNRAS, preprint (arXiv:2107.13038)

Krause E., Chang T.-C., Doré O., Umetsu K., 2013, ApJ, 762, L20

Lange J. U., Leauthaud A., Singh S., Guo H., Zhou R., Smith T. L., Cyr-Racine F.-Y., 2021, MNRAS, 502, 2074

Lanusse F., Starck J. L., Leonard A., Pires S., 2016, A\&A, 591, A2

Leauthaud A. et al., 2017, MNRAS, 467, 3024

Mackenzie R., Shanks T., Bremer M. N., Cai Y.-C., Gunawardhana M. L. P., Kovács A., Norberg P., Szapudi I., 2017, MNRAS, 470, 2328

Manzotti A., Dodelson S., 2014, Phys. Rev. D, 90, 123009

Marcos-Caballero A., Fernández-Cobos R., Martínez-González E., Vielva P., 2016, MNRAS, 460, L15

Melchior P., Sutter P. M., Sheldon E. S., Krause E., Wandelt B. D., 2014, MNRAS, 440, 2922

Morganson E. et al., 2018, PASP, 130, 074501

Nadathur S., Crittenden R., 2016, ApJ, 830, L19

Nadathur S., Hotchkiss S., Sarkar S., 2012, J. Cosmol. Astropart. Phys., 2012, 42

Nadathur S., Lavinto M., Hotchkiss S., Räsänen S., 2014, Phys. Rev. D, 90, 103510

Nadathur S., Hotchkiss S., Crittenden R., 2017, MNRAS, 467, 4067

Naidoo K., Benoit-Lévy A., Lahav O., 2016, MNRAS, 459, L71

Naidoo K., Benoit-Lévy A., Lahav O., 2017, MNRAS, 472, L65

Pandey S. et al., 2021, preprint (arXiv:2105.13545)

Planck Collaboration XXIII, 2014, A\&A, 571, A23

Planck Collaboration VI, 2020, A\&A, 641, A6

Pollina G., Hamaus N., Dolag K., Weller J., Baldi M., Moscardini L., 2017, MNRAS, 469, 787

Pollina G. et al., 2019, MNRAS, 487, 2836

Prat J. et al., 2021 preprint (arXiv:e-prints)

Raghunathan S., Nadathur S., Sherwin B. D., Whitehorn N., 2020, ApJ, 890, 168

Rassat A., Starck J. L., Dupé F. X., 2013, A\&A, 557, A32

Rees M. J., Sciama D. W., 1968, Nature, 217, 511

Ribli D., Pataki B. Á., Zorrilla Matilla J. M., Hsu D., Haiman Z., Csabai I., 2019, MNRAS, 490, 1843

Ross A. J. et al., 2020, MNRAS, 498, 2354

Rozo E. et al., 2016, MNRAS, 461, 1431

Rudnick L., Brown S., Williams L. R., 2007, ApJ, 671, 40

Rykoff E. S. et al., 2014, ApJ, 785, 104

Sachs R. K., Wolfe A. M., 1967, ApJ, 147, 73

Sánchez C. et al., 2017, MNRAS, 465, 746

Secco L. F. et al., 2021, preprint (arXiv:2105.13544)

Sevilla I. et al., 2011, preprint (arXiv:1109.6741)

Sevilla-Noarbe I. et al., 2021, ApJS, 254, 34

Sheldon E. S., Huff E. M., 2017, ApJ, 841, 24

Shimakawa R. et al., 2021, MNRAS, 503, 3896

Skrutskie M. F. et al., 2006, AJ, 131, 1163

Smith K. M., Huterer D., 2010, MNRAS, 403, 2

Springel V. et al., 2005, Nature, 435, 629

Szapudi I. et al., 2015, MNRAS, 450, 288

Takahashi R., Sato M., Nishimichi T., Taruya A., Oguri M., 2012, ApJ, 761, 152

Takahashi R., Hamana T., Shirasaki M., Namikawa T., Nishimichi T., Osato K., Shiroyama K., 2017, ApJ, 850, 24

Vielva P., 2010, Adv. Astron., 2010, 592094

Vielzeuf P. et al., 2021, MNRAS, 500, 464

Watson W. A. et al., 2014, MNRAS, 438, 412

Wright E. L. et al., 2010, AJ, 140, 1868

Zaroubi S., Hoffman Y., Fisher K. B., Lahav O., 1995, ApJ, 449, 446

Zhang R., Huterer D., 2010, Astropart. Phys., 33, 69 


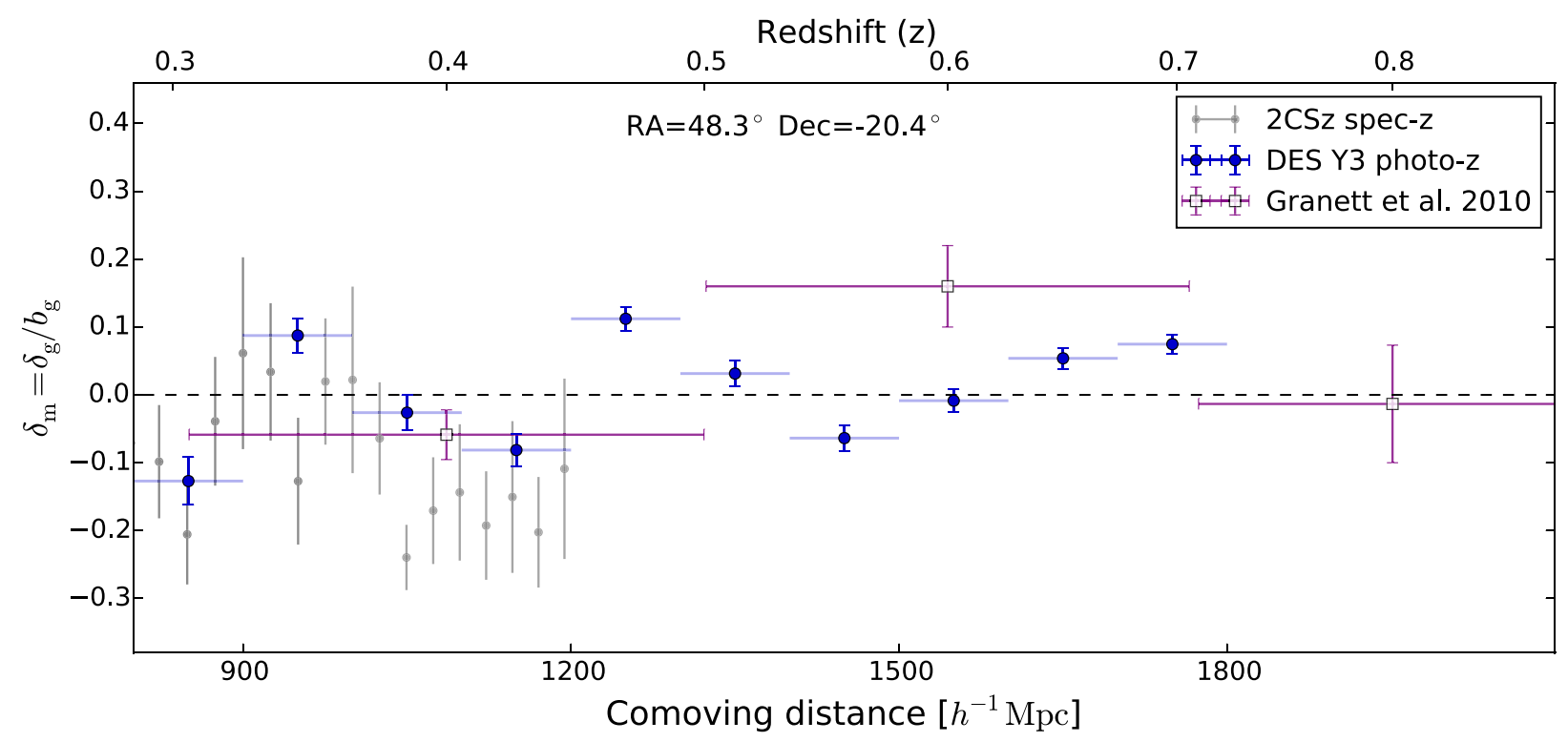

Figure A1. Extending the previously shown lower $z$ range, we compare line-of-sight matter density profiles at $0.3<z<0.9$ for different surveys of galaxies in the CS direction. As in Fig. 3, we converted galaxy density to matter density using the independently determined linear galaxy bias $\left(b_{\mathrm{g}}\right)$ values for each tracer data set. In good consistency with previous results by Granett et al. (2010) who conducted a dedicated photo- $z$ survey in the area, the DES results from redMaGiC galaxies show no evidence for significant voids or overdensities beyond the $z<0.3$ range where the Eridanus supervoid is observed.

Zürcher D., Fluri J., Sgier R., Kacprzak T., Refregier A., 2021, J. Cosmol. Astropart. Phys., 2021, 028

\section{APPENDIX: DENSITY PROFILE ANALYSIS}

Here we provide further details on our DES Y3 measurements of the high- $z$ matter density profile in the direction of the CS. In Fig. A1, we illustrate that the DES Y3 data shows no evidence for significant voids or overdensities at the $0.3<z<0.9$ range beyond the known underdensities at $z<0.3$ in Eridanus. These results are consistent the findings by Granett et al. (2010) and Bremer et al. (2010).

\footnotetext{
${ }^{1}$ Instituto de Astrofísica de Canarias (IAC), Calle Vía Láctea, E-38200 La Laguna, Tenerife, Spain

${ }^{2}$ Departamento de Astrofísica, Universidad de La Laguna (ULL), E-38206 La Laguna, Tenerife, Spain

${ }^{3}$ Laboratoire de Physique de l'Ecole Normale Supérieure, ENS, Université PSL, CNRS, Sorbonne Université, Université de Paris, 24 Rue Lhomond, F-75005 Paris, France

${ }^{4}$ Department of Physics and Astronomy, University College London, Gower Street, London WC1E 6BT, UK

${ }^{5}$ Institut de Física d'Altes Energies (IFAE), The Barcelona Institute of Science and Technology, Campus UAB, E-08193 Bellaterra, Spain

${ }^{6}$ Department of Physics and Astronomy, University of Pennsylvania, Philadelphia, PA 19104, USA

${ }^{7}$ Department of Astronomy and Astrophysics, University of Chicago, Chicago, IL 60637, USA

${ }^{8}$ Kavli Institute for Cosmological Physics, University of Chicago, Chicago, IL 60637, USA

${ }^{9}$ Universitäts-Sternwarte, Fakultät für Physik, Ludwig-Maximilians Universität München, Scheinerstr. 1, D-81679 München, Germany

${ }^{10}$ Institute of Cosmology and Gravitation, University of Portsmouth, Portsmouth PO1 3FX, UK

${ }^{11}$ Department of Physics, ETH Zurich, Wolfgang-Pauli-Strasse 16, CH-8093

Zurich, Switzerland
}

${ }^{12}$ Instituto de Fisica Teorica UAM/CSIC, Universidad Autonoma de Madrid, E-28049 Madrid, Spain

${ }^{13}$ Argonne National Laboratory, 9700 South Cass Avenue, Lemont, IL 60439, USA

${ }^{14}$ Kavli Institute for Particle Astrophysics and Cosmology, PO Box 2450, Stanford University, Stanford, CA 94305, USA

${ }^{15}$ Physics Department, 2320 Chamberlin Hall, University of WisconsinMadison, 1150 University Avenue Madison, WI 53706-1390, USA

${ }^{16}$ Department of Physics, Carnegie Mellon University, Pittsburgh, PA 15312, USA

${ }^{17}$ Laboratório Interinstitucional de e-Astronomia - LIneA, Rua Gal. José Cristino 77, Rio de Janeiro, RJ - 20921-400, Brazil

${ }^{18}$ Center for Astrophysical Surveys, National Center for Supercomputing Applications, 1205 West Clark St., Urbana, IL 61801, USA

${ }^{19}$ Department of Astronomy, University of Illinois at Urbana-Champaign, 1002 W. Green Street, Urbana, IL 61801, USA

${ }^{20}$ Department of Physics, Duke University Durham, NC 27708, USA

${ }^{21}$ Center for Cosmology and Astro-Particle Physics, The Ohio State University, Columbus, OH 43210, USA

${ }^{22}$ Jodrell Bank Center for Astrophysics, School of Physics and Astronomy, University of Manchester, Oxford Road, Manchester M13 9PL, UK

${ }^{23}$ Department of Astronomy, University of California, Berkeley, 501 Campbell Hall, Berkeley, CA 94720, USA

${ }^{24}$ Santa Cruz Institute for Particle Physics, Santa Cruz, CA 95064, USA

${ }^{25}$ Fermi National Accelerator Laboratory, PO Box 500, Batavia, IL 60510, USA

${ }^{26}$ Department of Physics, The Ohio State University, Columbus, OH 43210, USA

${ }^{27}$ Jet Propulsion Laboratory, California Institute of Technology, 4800 Oak Grove Dr., Pasadena, CA 91109, USA

${ }^{28}$ Department of Physics, Stanford University, 382 Via Pueblo Mall, Stanford, CA 94305, USA

${ }^{29}$ SLAC National Accelerator Laboratory, Menlo Park, CA 94025, USA

${ }^{30}$ Department of Physics, University of Oxford, Denys Wilkinson Building, Keble Road, Oxford OX1 3RH, UK

${ }^{31}$ Department of Astronomy, University of Geneva, ch. d'Écogia 16, CH-1290 Versoix, Switzerland 
${ }^{32}$ Department of Physics, University of Michigan, Ann Arbor, MI 48109, USA

${ }^{33}$ Department of Applied Mathematics and Theoretical Physics, University of Cambridge, Cambridge CB3 OWA, UK

${ }^{34}$ Perimeter Institute for Theoretical Physics, 31 Caroline St $N$, Waterloo, ON N2L 2Y5, Canada

${ }^{35}$ Instituto de Física Gleb Wataghin, Universidade Estadual de Campinas, Campinas, SP 13083-859, Brazil

${ }^{36}$ Centro de Investigaciones Energéticas, Medioambientales y Tecnológicas (CIEMAT), Av. Complutense, 40, E-28040 Madrid, Spain

${ }^{37}$ Brookhaven National Laboratory, Bldg 510, Upton, NY 11973, USA

${ }^{38}$ Institut d'Estudis Espacials de Catalunya (IEEC), E-08034 Barcelona, Spain

${ }^{39}$ Institute of Space Sciences (ICE, CSIC), Campus UAB, Carrer de Can Magrans, s/n, E-08193 Barcelona, Spain

${ }^{40}$ Max Planck Institute for Extraterrestrial Physics, Giessenbachstrasse, D85748 Garching, Germany

${ }^{41}$ Institute for Astronomy, University of Edinburgh, Edinburgh EH9 3HJ, UK

${ }^{42}$ Departamento de Física Matemática, Instituto de Física, Universidade de São Paulo, CP 66318, São Paulo, SP 05314-970, Brazil

${ }^{43}$ Instituto de Física Teórica, Universidade Estadual Paulista, R. Dr. Bento Teobaldo Ferraz, 271 - Várzea da Barra Funda, São Paulo - SP, 01140-070, Brazil

${ }^{44}$ CNRS, UMR 7095, Institut d'Astrophysique de Paris, F-75014 Paris, France ${ }^{45}$ Sorbonne Universités, UPMC Univ Paris 06, UMR 7095, Institut d'Astrophysique de Paris, F-75014 Paris, France

${ }^{46}$ Astronomy Unit, Department of Physics, University of Trieste, via Tiepolo 11, I-34131 Trieste, Italy

${ }^{47}$ INAF-Osservatorio Astronomico di Trieste, via G. B. Tiepolo 11, I-34143 Trieste, Italy

${ }^{48}$ Institute for Fundamental Physics of the Universe, Via Beirut 2, I-34014 Trieste, Italy
${ }^{49}$ Observatório Nacional, Rua Gal. José Cristino 77, Rio de Janeiro, RJ 20921-400, Brazil

${ }^{50}$ School of Mathematics and Physics, University of Queensland, Brisbane, QLD 4072, Australia

${ }^{51}$ Department of Physics, IIT Hyderabad, Kandi, Telangana 502285, India

${ }^{52}$ Institute of Theoretical Astrophysics, University of Oslo. PO Box 1029 Blindern, NO-0315 Oslo, Norway

${ }^{53}$ Department of Astronomy, University of Michigan, Ann Arbor, MI 48109, USA

${ }^{54}$ Institute of Astronomy, University of Cambridge, Madingley Road, Cambridge CB3 OHA, UK

${ }^{55}$ Kavli Institute for Cosmology, University of Cambridge, Madingley Road, Cambridge CB3 OHA, UK

${ }^{56}$ Center for Astrophysics, Harvard and Smithsonian, 60 Garden Street, Cambridge, MA 02138, USA

${ }^{57}$ Lowell Observatory, 1400 W Mars Hill Rd, Flagstaff, AZ 86001, USA

${ }^{58}$ Australian Astronomical Optics, Faculty of Science and Engineering, Macquarie University, Macquarie Park, NSW 2113, Australia

${ }^{59}$ George P. and Cynthia Woods Mitchell Institute for Fundamental Physics and Astronomy, and Department of Physics and Astronomy, Texas A\&M University, College Station, TX 77843, USA

${ }^{60}$ Department of Astrophysical Sciences, Princeton University, Peyton Hall, Princeton, NJ 08544, USA

${ }^{61}$ Institució Catalana de Recerca i Estudis Avançats, Barcelona, Spain

${ }^{62}$ Department of Physics and Astronomy, Pevensey Building, University of Sussex, Brighton BN1 9QH, UK

${ }^{63}$ School of Physics and Astronomy, University of Southampton, Southampton SO17 1BJ, UK

${ }^{64}$ Computer Science and Mathematics Division, Oak Ridge National Laboratory, Oak Ridge, TN 37831, USA

This paper has been typeset from a $\mathrm{T}_{\mathrm{E}} \mathrm{X} / \mathrm{L} \mathrm{T} \mathrm{E} \mathrm{X}$ file prepared by the author. 